\title{
Continental copepods (Crustacea: Hexanauplia) of Colombia: revision and additions to the inventory
}

\section{Copépodos (Crustacea: Hexanauplia) continentales de Colombia: revisión y adiciones al inventario}

\author{
Santiago Gaviria and Nelson Aranguren-Riaño
}

\begin{abstract}
We present the compilation of published and unpublished records of continental copepods of Colombia, as well as personal observations by the authors, yielding an additional list of 52 species and subspecies ( 7 calanoids, 20 cyclopoids, 25 harpacticoids). In addition to our former inventory (2007) of 69 species, the total number now reaches 121 taxa, increasing by $75 \%$ the known number of continental copepods. Freshwater taxa increased in 15 species and subspecies. The number of brackish species (and marine species collected in brackish environments), recorded from coastal lagoons and temporal offshore ponds reached 39 species and subspecies. Thirteen taxa with locus typicus in Colombia have been described since 2007. Between 2007 and 2018, thirty-nine departmental records were made, and 43 new habitat records were reported (not including the species recorded as new for the country). Parasitic copepods of fish reached six species. However, the number of species is expected to increase with the survey of poorly studied regions like the Amazon and the Eastern Plains, and habitats like groundwater, benthos of lakes and ponds, semiterrestrial environments and additional coastal lagoons.
\end{abstract}

Keywords. Biodiversity. Geographic distribution. Meiobenthos. Neotropical region. Zooplankton.

\section{Resumen}

Como resultado de la compilación de datos publicados y no publicados de copépodos continentales de Colombia, así como de observaciones personales de los autores, se estableció una lista adicional de 52 especies y subespecies (7 calanoideos, 20 cyclopoideos, 25 harpacticoideos). Junto con el inventario del año 2007 (69 taxa), el número actual alcanza 121 especies y subespecies, lo que representa un incremento del $75 \%$. El número de especies de agua dulce se incrementa en 15 especies y subespecies. El número de especies de aguas salobres (y de especies marinas recolectadas en ambientes salobres), reportadas en lagunas costeras y charcas cercanas a la costa, es de 39 especies y subespecies. Desde el año 2007, se describieron 13 taxones nuevos con locus typicus en Colombia. En el periodo comprendido entre el 2007 y el 2018 se registraron 39 especies en departamentos sin registros previos y 43 registros en biotopos no señalados para determinadas especies (sin considerar aquellas especies desconocidas anteriormente en Colombia). Se registraron seis especies de copépodos parásitos de peces. Sin embargo, con el estudio de regiones poco conocidas como el Amazonas y los Llanos Orientales, biotopos poco estudiados como las aguas subterráneas, el bentos de lagos y charcas, ambientes semiterrestres, así como nuevas lagunas costeras, es de esperar un aumento en el número de especies.

Palabras clave. Biodiversidad. Distribución geográfica. Meiobentos. Región Neotropical. Zooplancton. 


\section{Introduction}

The subclass Copepoda includes small crustaceans inhabiting almost every aquatic biotope, as well as semiterrestrial habitats such as mosses and humid forest soils. Copepods inhabit from deep-sea trenches up to high mountain lakes of the Andes, Mount Kenya and the Himalaya. The total number of copepods exceeds 11,300 accepted species and subspecies; together with nematodes they are the most abundant metazoans on Earth (Walter \& Boxshall, 2019). In continental waters, about 2500 species and subspecies are known, but this number increases when coastal lagoons with different salinity values are also considered. The body size of adult copepods ranges from 0.2 to $17 \mathrm{~mm}$, but most average 1-2 $\mathrm{mm}$. Copepods can be free-living, symbiotic as well as internal or external parasites of almost all major aquatic metazoans (Huys \& Boxshall, 1991). In freshwaters, parasitic copepods are found only in fish.

A checklist of the free-living copepods of continental waters of Colombia was published 12 years ago (Gaviria \& Aranguren, 2007), including 69 species and subspecies (14 Calanoida, 41 Cyclopoida, 14 Harpacticoida).

During the past years, the copepod fauna of new biotopes such as coastal lagoons, phytotelmata of the rain forest, Amazon floodplain lakes (varzea and igapó), freshwater lagoons (ciénagas), wetlands of the eastern Llanos, Andean lakes, ponds, wet mosses and reservoirs have been studied. Moreover, the results presented here include reports of parasitic copepods of fish (Lernaeidae and Ergasilidae) obtained from studies developed in the departments of Valle del Cauca, Meta and Magdalena.

Coastal lagoons were considered in our inventory due to their topotypical character with narrow connection to the sea. Because of their connection to the marine environment, they show a wide range of salinity. Estuaries like those from the Pacific coast are not considered in the inventory. Coastal ponds are morphologically isolated from the sea and can temporarily reach hypersalinity. Thus, the following groups with brackish or marine representatives are considered in the inventory: Calanoida (Acartiidae, Lucicutidae, Pseudodiaptomidae, Temoridae), Cyclopoida (Halicyclopinae, Kelleridae, Oithonidae, Apocyclops) and Harpacticoida (Ameiridae, Ectinosomatidae, Laophontidae, Metidae, Miraciidae, Tachididae, Tegastidae, Cletocamptus, Mesochra). The remaining groups considered are the following: Calanoida (Centropagidae, Diaptomidae), Cyclopoida (Cyclopinae, Eucyclopinae) and Harpacticoida (Canthocamptidae, Parastenocarididae).

Aims of this contribution are to elaborate a revision of the species richness of continental copepods of Colombia, compare their diversity in relation to the previous inventory (Gaviria \& Aranguren, 2007), indicate orders, families, genera and species represented in the country, list the records of the copepod species in the different departments and biotopes, compare the species richness of the Colombian genera in relation to the Neotropical Region, and indicate the world distribution of the Colombian species. Finally, we propose points of future research in order to fill the gaps of the knowledge of diversity of the continental copepods of Colombia.

\section{Materials and methods}

The list of species presented here is the result of a critical compilation of published and unpublished records that appeared after 2007, and of personal observations of the authors and colleagues. Unpublished records are those indicated in Aranguren (2014) and obtained from the study of zooplankton of Amazonian lakes (varzea type: Yahuarcaca, Tarapoto and El Correo; igapó type: Zacambú), Andean lakes (Tota, Fúquene, Iguaque and Guatavita) and Caribbean ciénagas (Ayapel, Momil, Purísima and Vipis). Part of this information was published in Aranguren et al. (2011).

A synopsis of the families, genera, species and subspecies listed for the country until 2007 and until 2018 is shown in Table 2. For comparative purposes, the corresponding number of species and subspecies per genus occurring in the Neotropical region is included. 
The published references include species of free-living and parasitic copepods from continental biotopes and coastal lagoons and ponds, recorded during different types of studies, as follows:

1) Zooplankton during limnological studies of Amazonian lakes (Aranguren-Riaño et al., 2011), wetlands of the Orinoco basin (five lakes near the Orinoco River) (RiveraRondón et al., 2010), Andean lakes (Aranguren-Riaño et al., 2011) and reservoirs (Villabona-González et al., 2007, 2015; Aranguren-Riaño \& Monroy-González, 2014) and Caribbean ciénagas (Gallo-Sánchez et al., 2009; Álvarez, 2010; Aranguren-Riaño et al. 2011; Villabona et al., 2011; Jaramillo-Londoño \& Aguirre-Ramírez, 2012).

2) Benthic harpacticoids collected in phytotelmata of the rain forest during taxonomic studies (Gaviria \& Defaye, 2012), and in an Andean lake and a pond of the páramo region during taxonomic and phylogenetic studies (Laguna de Buitrago, Chingaza; pond near Laguna de San Rafael, Puracé) (Gaviria \& Defaye, 2012, 2015, 2017a, 2017b).

3) Limnetic and benthic species from coastal lagoons and temporary ponds of the Caribbean region during studies of taxonomy and biodiversity: the investigated coastal lagoons were Laguna del Navío Quebrado (La Guajira) (Fuentes-Reinés \& Gómez, 2014; Fuentes-Reinés \& Suárez-Morales, 2014a, 2014b, 2015; Suárez-Morales \& Fuentes-Reinés, 2014, 2015a, 2015b, 2015c) and Ciénaga Grande de Santa Marta (Magdalena) (Fuentes Reinés et al.,2013; Fuentes-Reinés \& Zoppi de Roa, 2013a, 2013b; Fuentes-Reinés \& Suárez-Morales, 2018; Fuentes-Reinés et al., 2018). The temporary ponds located in the Magdalena department are located in Pozos Colorados (Gómez et al., 2017) and Puebloviejo (Fuentes-Reinés et al., 2015).

4) Parasitic copepods and their fish hosts from various rivers and a coastal lagoon, studied by Cressey \& Colette (1970) (mouth of Dagua River, Valle del Cauca), Thatcher (1984) (Pital River, Valle del Cauca), Thatcher (2000) (Meta River, Meta), Fuentes-Reinés et al. (2012) (southern Ciénaga Grande de Santa Marta, Magdalena), Sarmiento \& Rodríguez (2013) and Muriel-Hoyos et al. (2015) (Vichada River, Meta).
New records of species for Colombia as well as new records of already known species are listed, indicating their overall distribution, their presence in the different Colombian departments and habitats, and the corresponding bibliographic references.

The records of personal observations (SG - S. Gaviria; NA - N. Aranguren; JM - J. Molina; DD - D. Defaye, DB - D. Baribwegure) are based on samples obtained at the following localities and years:

1. Amazonas: Laguna de Tarapacá, near Rio Putumayo, SG (2001). Laguna de Yahuarcaca, Laguna Zacambú, Laguna El Correo and Laguna Tarapoto, NA (2007).

2. Antioquia: Microestación, Campus Universidad de Antioquia, Medellín, SG (1999). Reservoir La Fé, SG (1999); Reservoir Porce II, SG (1999); Reservoir Riogrande II, SG (2001); lake at fishfarm Gaiteros, Sopetrán, SG (2001); Lake “Dos Lagos", Carmen de Viboral, SG (2001); Lake Cerro del Padre Amaya, Palmitas, SG (2001); Lake Piedras Blancas, Guarne, SG (1999); Ciénaga Vallecitos, Caucasia, SG (1999).

3. Boyacá: Laguna de Iguaque, SG (2010); wet moss páramo de Cómbita, SG \& DD (2016); Laguna Verde, páramo de Pisba, NA \& JM, 2018; Laguna de Socha, Laguna Peña Negra and Laguna Los Fríos NA (2016).

4. Cesar: Ciénaga de Zapatosa, SG \& DB (1999).

5. Chocó: Ciénaga de Tumaradó and Ciénaga de Perancho, SG (2000).

6. Córdoba: Ciénaga de Ayapel, SG 1999; Ciénaga de Betancí, SG 2002; Ciénaga de Lorica, SG (2002).

7. Cundinamarca: Fishpond in La Mesa, NA (1994).

8. Magdalena: Ciénaga de Pijiño, SG (1999).

9. Meta: Laguna Mateyuca, Puerto López, SG (1999).

10. Tolima: Ciénaga de Guarinocito, NA (2007). 
Information about the overall distribution of the freshwater species was extracted from Dussart \& Defaye (2002, 2006), Defaye \& Dussart (2011), FuentesReinés et al. (2013) and Perbiche-Neves et al. (2014). The distribution of planktonic species from brackish water follows Razouls et al. (2005-2018) and of benthic species of brackish water according to Fuentes-Reinés \& SuárezMorales (2013, 2015, 2018), Fuentes-Reinés \& Gómez (2014), Fuentes-Reinés \& Suárez-Morales (2014a, 2014b), Fuentes-Reinés et al. $(2013 b, 2015,2018)$ and Gómez et al. (2017). Taxonomy follows Walter \& Boxshall (2019) (http://www.marinespecies.org/copepoda).

\section{Results}

The taxonomic list (Table 1) presents the new records of copepods in continental waterbodies, including 15 families, 25 genera and 52 species and subspecies not recorded in a previous Colombian inventory. The current number of copepods recorded in Colombia comprises 121 taxa (21 calanoids, 61 cyclopoids and 39 harpacticoids), including taxa from coastal lagoons and ponds, as well as parasitic species.

Besides the new records for the country, we report the occurrence of 39 species in new departments, 43 new habitat records and seven new bibliographic references (Table 1). Eight of the 32 Colombian departments, i.e. Arauca, Caldas, Caquetá, Casanare, Guaviare, Putumayo, Quindío and Vaupés, lack reports of copepods. Most records are from Magdalena (42), La Guajira (34) and Cundinamarca (27).

Seventy-four percent of the species are distributed in lowland waterbodies, $17 \%$ in highland regions (from $2000 \mathrm{~m}$ a. s. 1.) and $9 \%$ in both regions. Finally, the known altitudinal or geographic range of distribution of 29 species has increased.

Table 1. Taxonomic list of the species and subspecies of copepods reported after 2007 in continental waterbodies, semiterrestrial biotopes, coastal lagoons and temporal offshore ponds of Colombia. Taxa known before 2007 but with expanded distribution in new departments, increase of altitude range and habitats not indicated before, are included. Expansion of altitudinal range, departments and habitats are indicated in bold.

\begin{tabular}{|c|c|c|c|c|c|c|}
\hline Taxon & $\begin{array}{l}\text { World } \\
\text { distribution }\end{array}$ & $\begin{array}{l}\text { Distribution } \\
\text { in Colombia }\end{array}$ & $\begin{array}{l}\text { Altitude } \\
\text { (m a.s. 1.) }\end{array}$ & Habitat & $\begin{array}{l}\text { Reference } \\
\text { collection }\end{array}$ & Bibliographic reference \\
\hline \multicolumn{7}{|l|}{$\begin{array}{l}\text { ORDER CALANOI- } \\
\text { DA }\end{array}$} \\
\hline \multicolumn{7}{|l|}{$\begin{array}{l}\text { FAMILY ACARTII- } \\
\text { DAE }\end{array}$} \\
\hline $\begin{array}{l}\text { Acartia (Odontocartia) } \\
\text { lilljeborgi Giesbrecht, } \\
1889\end{array}$ & $\begin{array}{l}\text { Gulf-Mex Car Pac } \\
\text { COL }\end{array}$ & lag & 0 & $\begin{array}{l}\text { coas-lag } \\
\text { estua pel }\end{array}$ & UARC & $\begin{array}{l}\text { Fuentes-Reinés \& Suárez-Mo- } \\
\text { rales, } 2015\end{array}$ \\
\hline $\begin{array}{l}\text { Acartia (Acanthacartia) } \\
\text { tonsa Dana } 1849\end{array}$ & Cosm COL & lag mar & 0 & $\begin{array}{l}\text { coas-lag } \\
\text { euryhal pel }\end{array}$ & UARC & $\begin{array}{l}\text { Pearse, 1915; Fuentes-Reinés } \\
\text { et al., 2013; Fuentes-Reinés \& } \\
\text { Suárez-Morales, } 2015\end{array}$ \\
\hline \multicolumn{7}{|l|}{$\begin{array}{l}\text { FAMILY CENTROP- } \\
\text { AGIDAE }\end{array}$} \\
\hline $\begin{array}{l}\text { Boeckella gracilis Da- } \\
\text { day, } 1902\end{array}$ & $\begin{array}{l}\text { ARG BOL CHL } \\
\text { COL ECU PER }\end{array}$ & $\begin{array}{l}\text { cun boy met } \\
\text { nar }\end{array}$ & $\begin{array}{l}3000- \\
3800\end{array}$ & lak pn & NHMW & $\begin{array}{l}\text { Gaviria, 1989; Aranguren-Riaño } \\
\text { et al., 2011; Aranguren-Riaño, } \\
2014\end{array}$ \\
\hline $\begin{array}{l}\text { FAMILY DIAPTOMI- } \\
\text { DAE }\end{array}$ & & & & & & \\
\hline
\end{tabular}




\begin{tabular}{|c|c|c|c|c|c|c|}
\hline $\begin{array}{l}\text { Arctodiaptomus dorsalis } \\
\text { (Marsh, 1907) }\end{array}$ & $\begin{array}{l}\text { Amer COL CRI } \\
\text { CUB GUF MEX } \\
\text { NIC PAN Hisp PRI } \\
\text { USA VEN }\end{array}$ & $\begin{array}{l}\text { ant cau cho } \\
\text { cor nsan san }\end{array}$ & $0-2200$ & $\begin{array}{l}\text { cie res riv } \\
\text { pel pn }\end{array}$ & UIS & $\begin{array}{l}\text { Buitrago, 1998; SG. pers. obs. } \\
\text { 1999, 2001; Reid, 2007; Villabo- } \\
\text { na-González et al., 2007, 2015; } \\
\text { Aranguren-Riaño et al., 2011; } \\
\text { Gaviria, 1989; Aranguren-Ria- } \\
\text { ño, } 2014\end{array}$ \\
\hline $\begin{array}{l}\text { Colombodiaptomus } \\
\text { brandorffi Gaviria, } 1989\end{array}$ & COL & boy cun & $\begin{array}{l}2900- \\
3730\end{array}$ & $\begin{array}{l}\text { lak pel pn } \\
\text { res }\end{array}$ & NHMW & $\begin{array}{l}\text { Gaviria, 1989; SG pers.obs. } \\
\text { 2010; Aranguren-Riaño et al., } \\
\text { 2011; Aranguren-Riaño, } 2014\end{array}$ \\
\hline $\begin{array}{l}\text { Dactylodiaptomus pear- } \\
\text { sei (Whright, 1927) }\end{array}$ & COL BRA VEN & ama & 300 & lak & & $\begin{array}{l}\text { Aranguren-Riaño et al., 2011, } \\
2014\end{array}$ \\
\hline $\begin{array}{l}\text { Dasydiaptomus corona- } \\
\text { tus (G.O. Sars, 1901) }\end{array}$ & $\begin{array}{l}\text { ARG BRA COL } \\
\text { VEN }\end{array}$ & vich & 300 & lak & & Rivera-Rondón et al., 2010 \\
\hline $\begin{array}{l}\text { Notodiaptomus conife- } \\
\text { roides (Wright, 1927) }\end{array}$ & $\begin{array}{l}\text { ARG BOL BRA } \\
\text { COL PRY }\end{array}$ & cor mag tol & $0-200$ & $\begin{array}{l}\text { backw cie } \\
\text { pel }\end{array}$ & & $\begin{array}{l}\text { N.A. \& S.G. pers.obs. } 1999 \\
\text { (2007); Álvarez, 2010; Villabo- } \\
\text { na-González et al., 2011; Jara- } \\
\text { millo-Londoño \& Aguirre-Ra- } \\
\text { mírez, 2012; Aranguren-Riaño, } \\
2014\end{array}$ \\
\hline $\begin{array}{l}\text { Notodiaptomus dilatatus } \\
\text { Dussart, } 1984\end{array}$ & BRA COL VEN & guai vich & 300 & lak pel riv & $\mathrm{MNHN}$ & $\begin{array}{l}\text { Dussart, 1984; Rivera-Rondón } \\
\text { et al., } 2010\end{array}$ \\
\hline $\begin{array}{l}\text { Notodiaptomus echina- } \\
\text { tus (Lowndes, 1934) } \\
\end{array}$ & $\begin{array}{l}\text { ARG BRA COL } \\
\text { GUF PRY VEN }\end{array}$ & guai vich & 300 & lak pel riv & $\mathrm{MNHN}$ & $\begin{array}{l}\text { Dussart, 1984; Rivera-Rondón } \\
\text { et al., } 2010\end{array}$ \\
\hline $\begin{array}{l}\text { Notodiaptomus henseni } \\
\text { (Dahl, 1894) }\end{array}$ & $\begin{array}{l}\text { BRA COL GUF } \\
\text { PRY VEN }\end{array}$ & $\begin{array}{l}\text { boy cor guai } \\
\text { vich }\end{array}$ & $\begin{array}{l}100- \\
2790\end{array}$ & $\begin{array}{l}\text { cie lak riv } \\
\text { pel }\end{array}$ & & $\begin{array}{l}\text { Dussart, 1984; Cicchino et al., } \\
\text { 1989; Villabona-González et al., } \\
\text { 2011; Aranguren-Riaño, 2014; } \\
\text { JM \& NA, pers. obs. } 2018\end{array}$ \\
\hline $\begin{array}{l}\text { Notodiaptomus linus } \\
\text { (Brandorff, 1973) }\end{array}$ & BRA COL & ama & $0-300$ & lak pel & & $\begin{array}{l}\text { Aranguren-Riaño et al., 2011; } \\
\text { Aranguren-Riaño, } 2014\end{array}$ \\
\hline $\begin{array}{l}\text { Notodiaptomus maracai- } \\
\text { bensis Kiefer, } 1956\end{array}$ & COL VEN & $\begin{array}{l}\text { atl ces cho } \\
\text { cor mag }\end{array}$ & $0-200$ & cie pel & SMNK & $\begin{array}{l}\text { Kiefer, 1956; SG pers. obs. 1999; } \\
\text { Álvarez, 2010; Aranguren-Ria- } \\
\text { ño et al., 2011; Villabona-Gonzá- } \\
\text { lez et al., 2011; Aranguren-Ria- } \\
\text { ño, } 2014\end{array}$ \\
\hline $\begin{array}{l}\text { Notodiaptomus similli- } \\
\text { mus Cicchino, Santos } \\
\text { Silva \& Robertson, } \\
2001 \\
\end{array}$ & BRA COL VEN & cor guai met & 300 & $\begin{array}{l}\text { cie lak pel } \\
\text { riv }\end{array}$ & & $\begin{array}{l}\text { Dussart, } 1984 \text { (as N. coniferoi- } \\
\text { des); Cicchino et al., 2001; SG } \\
\text { pers. obs. } 1999 \text { (2007); Villabo- } \\
\text { na-González et al., } 2011\end{array}$ \\
\hline $\begin{array}{l}\text { Prionodiaptomus colom- } \\
\text { biensis Thiébaud, } 1912\end{array}$ & $\begin{array}{l}\text { BRA COL SLV } \\
\text { GTM HND MEX } \\
\text { NIC PER PAN USA } \\
\text { VEN }\end{array}$ & $\begin{array}{l}\text { atl boy cor } \\
\text { mag cun }\end{array}$ & $0-2600$ & $\begin{array}{l}\text { cie lak pel } \\
\text { sw }\end{array}$ & NHMW & Álvarez, 2010; Gaviria, 1989 \\
\hline $\begin{array}{l}\text { Rhacodiaptomus ring- } \\
\text { ueleti Cicchino \& Dus- } \\
\text { sart, } 1991\end{array}$ & COL VEN & guai vich & 300 & $\begin{array}{l}\text { lak pel pn } \\
\text { riv }\end{array}$ & & $\begin{array}{l}\text { Cicchino \& Dussart, 1991; Rive- } \\
\text { ra-Rondón et al., } 2010\end{array}$ \\
\hline \multicolumn{7}{|l|}{$\begin{array}{l}\text { FAMILY LUCICUT- } \\
\text { IDAE }\end{array}$} \\
\hline $\begin{array}{l}\text { Lucicutia flavicornis } \\
\text { (Claus, 1863) }\end{array}$ & $\begin{array}{l}\text { Atl Ind Med Pac } \\
\text { COL }\end{array}$ & mag & 0 & $\begin{array}{l}\text { coas-lag } \\
\text { pel }\end{array}$ & UARC & Fuentes-Reinés et al., 2013 \\
\hline $\begin{array}{l}\text { FAMILY PSEUDODI- } \\
\text { APTOMIDAE }\end{array}$ & & & & & & \\
\hline
\end{tabular}




\begin{tabular}{|c|c|c|c|c|c|c|}
\hline $\begin{array}{l}\text { Pseudodiaptomus mars- } \\
\text { hi Wright S.,1936 }\end{array}$ & COL CRI BLZ MEX & lag mag & 0 & $\begin{array}{l}\text { coas-lag } \\
\text { euryhal de } \\
\text { epib veg }\end{array}$ & UARC & $\begin{array}{l}\text { Fuentes-Reinés et al., 2013; Fu- } \\
\text { entes-Reinés \& Suárez-Morales, } \\
2015\end{array}$ \\
\hline \multicolumn{7}{|l|}{$\begin{array}{l}\text { FAMILY TEMORI- } \\
\text { DAE }\end{array}$} \\
\hline $\begin{array}{l}\text { Temora turbinata } \\
\text { (Dana, 1849) }\end{array}$ & $\begin{array}{l}\text { Atl Indo-Pac Trop } \\
\text { Subtrop COL }\end{array}$ & lag & 0 & $\begin{array}{l}\text { coas-lag } \\
\text { brack pel }\end{array}$ & UARC & $\begin{array}{l}\text { Fuentes-Reinés \& Suárez-Mo- } \\
\text { rales, } 1915\end{array}$ \\
\hline \multicolumn{7}{|l|}{$\begin{array}{l}\text { ORDER CYCLOPOI- } \\
\text { DA }\end{array}$} \\
\hline \multicolumn{7}{|l|}{$\begin{array}{l}\text { FAMILY CORYCA- } \\
\text { EIDAE }\end{array}$} \\
\hline $\begin{array}{l}\text { Corycaeus (Corycaeus) } \\
\text { clausi F. Dahl, } 1894\end{array}$ & $\begin{array}{l}\text { Atl Ind Pac Trop } \\
\text { Subtrop }\end{array}$ & lag & 0 & $\begin{array}{l}\text { coas-lag } \\
\text { brackw pel }\end{array}$ & UARC & $\begin{array}{l}\text { Fuentes-Reinés \& Suárez-Mo- } \\
\text { rales, } 2015\end{array}$ \\
\hline $\begin{array}{l}\text { FAMILY CYCLOPI- } \\
\text { DAE }\end{array}$ & & & & & & \\
\hline
\end{tabular}

SUBFAMILY CYCLO-

PINAE

\begin{tabular}{|c|c|c|c|c|c|c|}
\hline $\begin{array}{l}\text { Apocyclops panamensis } \\
\text { (Marsh, 1913) }\end{array}$ & Amer COL USA & $\begin{array}{l}\text { cho lag mag } \\
\text { sap }\end{array}$ & 0 & $\begin{array}{l}\text { cav co- } \\
\text { ast-lag } \\
\text { euryhal }\end{array}$ & MBUCV & $\begin{array}{l}\text { Reid, 1988; Petkovski, 1988; } \\
\text { Fuentes-Reinés et al., 2013; Fu- } \\
\text { entes-Reinés \& Suárez-Morales, } \\
2015\end{array}$ \\
\hline $\begin{array}{l}\text { Mesocyclops brasilianus } \\
\text { Kiefer, } 1933\end{array}$ & $\begin{array}{l}\text { BRA CAN? COL } \\
\text { GTM HND VEN }\end{array}$ & $\begin{array}{l}\text { ama ant cal } \\
\text { cho cor mag } \\
\text { nar }\end{array}$ & $0-1500$ & $\begin{array}{l}\text { cie coas-lag } \\
\text { mm-pn }\end{array}$ & $\begin{array}{l}\text { USNM, } \\
\text { UARC }\end{array}$ & $\begin{array}{l}\text { Gallo-Sánchez et al., } 2009 \\
\text { (as M. venezolanus); Alvarez, } \\
2010 \text { (as M. venezolanus) Reid, } \\
\text { 1988; Gaviria, 1994; Aranguren, } \\
\text { 1998. Fuentes-Reinés et al. } \\
\text { 2013; Gaviria E Aranguren, } \\
2000 \text { (2007) (as M. venezolanus); } \\
\text { Aranguren-Riaño et al., } 2011 \text { \& } \\
\text { Aranguren-Riaño, } 2014 \text { (as M. } \\
\text { venezolanus); per. obs. JM \& } \\
\text { NA } 2016\end{array}$ \\
\hline $\begin{array}{l}\text { Mesocyclops ellipticus } \\
\text { Kiefer, } 1936\end{array}$ & $\begin{array}{l}\text { ARG COL CUB } \\
\text { BRA GUF PRY } \\
\text { VEN }\end{array}$ & lag mag & 0 & $\begin{array}{l}\text { coas-lag-fw } \\
\text { veg }\end{array}$ & $\begin{array}{l}\text { MBUCV } \\
\text { UARC }\end{array}$ & $\begin{array}{l}\text { Fuentes-Reinés et al., 2013; Fu- } \\
\text { entes-Reinés \& Suárez-Morales, } \\
2015\end{array}$ \\
\hline $\begin{array}{l}\text { Mesocyclops longisetus } \\
\text { (Thiébaud, 1912) }\end{array}$ & $\mathrm{Am}$ & $\begin{array}{l}\text { ama ant cor } \\
\text { cun mag }\end{array}$ & $0-2600$ & $\begin{array}{l}\text { coas-lag ig } \\
\text { lak lit pl res } \\
\text { riv veg }\end{array}$ & MBUCV & $\begin{array}{l}\text { Gaviria 1988; SG pers. obs. } \\
\text { 1999; NA pers. obs. 2007; } \\
\text { Fuentes-Reinés et al., 2013; } \\
\text { Aranguren-Riaño et al., 2011; } \\
\text { Aranguren-Riaño, 2014; Villa- } \\
\text { bona-González et al., } 2015\end{array}$ \\
\hline $\begin{array}{l}\text { Mesocyclops meridianus } \\
\text { (Kiefer, 1926) }\end{array}$ & $\begin{array}{l}\text { ARG BOL BRA } \\
\text { COL ECU lan PRY } \\
\text { PER VEN URY }\end{array}$ & $\begin{array}{l}\text { ama cun } \\
\text { guai }\end{array}$ & $100-500$ & est lak riv & & $\begin{array}{l}\text { Dussart, 1984; NA pers. obs. } \\
\text { 2007; Aranguren-Riaño et al., } \\
\text { 2011; Aranguren-Riaño, } 2014\end{array}$ \\
\hline $\begin{array}{l}\text { Metacyclops leptopus } \\
\text { leptopus (Kiefer, 1927) }\end{array}$ & $\begin{array}{l}\text { COL BOL ECU } \\
\text { PER }\end{array}$ & ant cun mag & $\begin{array}{l}2270- \\
3500\end{array}$ & $\begin{array}{l}\text { lak pn res } \\
\text { sw }\end{array}$ & & $\begin{array}{l}\text { Löffler, 1972; Gaviria, 1988; } \\
\text { Villabona-González et al., 2015; } \\
\text { Torres \& Rylander, } 2016\end{array}$ \\
\hline $\begin{array}{l}\text { Metacyclops leptopus } \\
\text { totaensis Reid, Molina } \\
\text { Arévalo \& Fukushi- } \\
\text { ma, } 1990\end{array}$ & COL & cund boy & $\begin{array}{l}3000- \\
3700\end{array}$ & lag pel & USNM & $\begin{array}{l}\text { Reid et al., 1990; Aranguren \& } \\
\text { Andrade, 2003; Aranguren-Ria- } \\
\text { ño et al., 2011; Aranguren-Ria- } \\
\text { ño, } 2014\end{array}$ \\
\hline
\end{tabular}




\begin{tabular}{|c|c|c|c|c|c|c|}
\hline $\begin{array}{l}\text { Metacyclops mendoci- } \\
\text { nus (Wierzejski, 1892) }\end{array}$ & $\begin{array}{l}\text { ARG BOL BRA } \\
\text { CHL COL ECU } \\
\text { GUF HTI NIC PRI } \\
\text { PRY PER URY } \\
\text { VEN }\end{array}$ & $\begin{array}{l}\text { ant cun san } \\
\text { sap }\end{array}$ & $0-2600$ & $\begin{array}{l}\text { est gw lak } \\
\text { lit pn res } \\
\text { tpl }\end{array}$ & & $\begin{array}{l}\text { Thiébaud, 1912; Petkovski, } \\
\text { 1998; Aranguren-Riaño et al., } \\
\text { 2011; Aranguren, } 2014\end{array}$ \\
\hline $\begin{array}{l}\text { Microcyclops anceps } \\
\text { anceps (Richard, 1897) }\end{array}$ & S-Amer & $\begin{array}{l}\text { ama ant cor } \\
\text { cun lag mag } \\
\text { vich }\end{array}$ & $0-1500$ & $\begin{array}{l}\text { cie coas-est } \\
\text { lag ig lak } \\
\text { lit pn sw }\end{array}$ & UARC & $\begin{array}{l}\text { NA pers. obs. 1994, 2007; SG } \\
\text { pers. obs. 1999; Alvarez, 2010; } \\
\text { Rivera-Rondón et al., 2010; } \\
\text { Aranguren-Riaño et al., 2011; } \\
\text { Fuentes-Reinés et al., 2013; } \\
\text { Fuentes-Reinés, 2015; Arangu- } \\
\text { ren-Riaño, } 2014\end{array}$ \\
\hline $\begin{array}{l}\text { Microcyclops anceps } \\
\text { pauxensis Herbst, } 1962\end{array}$ & BRA COL VEN & lag mag & 0 & $\begin{array}{l}\text { coas-lag lit } \\
\text { veg }\end{array}$ & $\begin{array}{l}\text { MBUCV, } \\
\text { UARC }\end{array}$ & $\begin{array}{l}\text { Fuentes-Reinés et al., 2013; Fu- } \\
\text { entes-Reinés \& Suárez-Morales, } \\
2015\end{array}$ \\
\hline $\begin{array}{l}\text { Microcyclops ceibaiensis } \\
\text { (Marsh, 1919) }\end{array}$ & Neotr & $\begin{array}{l}\text { cor lag mag } \\
\text { vich }\end{array}$ & $0-300$ & $\begin{array}{l}\text { cie coas-lag } \\
\text { lak lit veg }\end{array}$ & $\begin{array}{l}\text { MBUCV, } \\
\text { UARC }\end{array}$ & $\begin{array}{l}\text { SG pers. obs.1999 (2007); Álva- } \\
\text { rez, 2010; Rivera-Rondón et } \\
\text { al.,2010; Fuentes-Reinés et al., } \\
\text { 2013; Fuentes-Reinés, } 2015\end{array}$ \\
\hline $\begin{array}{l}\text { Microcyclops dubitabilis } \\
\text { Kiefer, } 1934\end{array}$ & Neotr USA-fl & $\begin{array}{l}\text { ant atl cor } \\
\text { cun mag nar }\end{array}$ & $0-2600$ & $\begin{array}{l}\text { cie co- } \\
\text { as-lag lak } \\
\text { lit pn res } \\
\text { sw veg }\end{array}$ & UARC & $\begin{array}{l}\text { SG \& NA pers. obs. } 1999 \text { (2007) } \\
\text { (as M. dubitabilis E M. alius); } \\
\text { Alvarez, 2010; Reid, } 1988 \text { (as M. } \\
\text { alius); Villabona-González et al., } \\
2011 \text { (as M. alius); Fuentes-Rei- } \\
\text { nés et al., } 2013\end{array}$ \\
\hline $\begin{array}{l}\text { Neutrocyclops brevifur- } \\
\text { ca (Lownes, 1934) }\end{array}$ & $\begin{array}{l}\text { ARG COL BRA } \\
\text { GTM MEX PRY } \\
\text { VEN }\end{array}$ & atl cor mag & $0-100$ & $\begin{array}{l}\text { coas-lag lit } \\
\text { SW veg }\end{array}$ & $\begin{array}{l}\text { MBUCV } \\
\text { SMNK }\end{array}$ & $\begin{array}{l}\text { Kiefer, 1956; SG pers.obs. } 1999 \\
\text { (2007); Álvarez, 2010; Fuen- } \\
\text { tes-Reinés et al., } 2013\end{array}$ \\
\hline $\begin{array}{l}\text { Thermocyclops crassus } \\
\text { (Fischer, 1853) ? }\end{array}$ & Cosm & ama cor & $0-300$ & cie var & & $\begin{array}{l}\text { Arangure-Riaño et al., 2011; } \\
\text { Aranguren-Riaño, 2014; (could } \\
\text { be T. decipiens) }\end{array}$ \\
\hline $\begin{array}{l}\text { Thermocyclops decipiens } \\
\text { (Kiefer, 1929) }\end{array}$ & Pantr & $\begin{array}{l}\text { ama ant atl } \\
\text { boj cal cor } \\
\text { cun hui lag } \\
\text { mag nar tol }\end{array}$ & $0-2000$ & $\begin{array}{l}\text { coast-lag- } \\
\text { fw lak pel } \\
\text { pn res sw } \\
\text { veg }\end{array}$ & $\begin{array}{l}\text { SMNK } \\
\text { UARC }\end{array}$ & $\begin{array}{l}\text { Kiefer, 1956; Aranguren, 1998, } \\
\text { 2014; Villabona-González-Gon- } \\
\text { zález et al., 2007; Alvarez, } \\
\text { 2010; Aranguren-Riaño et al., } \\
\text { 2011; Villabona-González et } \\
\text { al., 2011; Jaramillo-Londoño \& } \\
\text { Aguirre-Ramitez, 2012; Aran- } \\
\text { guren-Riaño, 2014; Arangu- } \\
\text { ren-Riaño \& Monroy-González, } \\
\text { 2014; Fuentes-Reinés \& Suá- } \\
\text { rez-Morales, } 2015\end{array}$ \\
\hline $\begin{array}{l}\text { Thermocyclops minutus } \\
\text { (Lowndes, 1934) }\end{array}$ & $\begin{array}{l}\text { ARG BRA COL } \\
\text { PRY VEN }\end{array}$ & cor vich & 300 & cie lak & & $\begin{array}{l}\text { Alvarez, 2010; Rivera Rondón } \\
\text { et al., } 2010\end{array}$ \\
\hline $\begin{array}{l}\text { Thermocyclops tenuis } \\
\text { (Marsh, 1920) }\end{array}$ & Amer & $\begin{array}{l}\text { ces cor mag } \\
\text { ama }\end{array}$ & $0-100$ & cie var & & $\begin{array}{l}\text { DB \& SG pers. obs. 2007; Álva- } \\
\text { rez, 2010; Aranguren, } 2014\end{array}$ \\
\hline \multicolumn{7}{|l|}{$\begin{array}{l}\text { SUBFAMILY EUCYC- } \\
\text { LOPINAE }\end{array}$} \\
\hline $\begin{array}{l}\text { Ectocyclops rubescens } \\
\text { Brady, } 1904\end{array}$ & Pantr & atl nar mag & $0-100$ & $\begin{array}{l}\text { ben co- } \\
\text { as-lag lak } \\
\text { pn veg }\end{array}$ & $\begin{array}{l}\text { SMNK } \\
\text { UARC } \\
\text { USNM }\end{array}$ & $\begin{array}{l}\text { Kiefer, 1956; Reid, 1988; Fuen- } \\
\text { tes-Reinés et al., } 2013\end{array}$ \\
\hline
\end{tabular}




\begin{tabular}{|c|c|c|c|c|c|c|}
\hline $\begin{array}{l}\text { Ectocyclops phaleratus } \\
\text { (Koch, 1838) }\end{array}$ & Cosm & cun mag nar & $0-2600$ & $\begin{array}{l}\text { ben co- } \\
\text { as-lag lak } \\
\text { sw pn veg } \\
\end{array}$ & $\begin{array}{l}\text { UARC } \\
\text { USNM }\end{array}$ & $\begin{array}{l}\text { Thiébaud, 1912; Reid, 1988; } \\
\text { Fuentes-Reinés et al., } 2013\end{array}$ \\
\hline $\begin{array}{l}\text { Eucyclops serrulatus } \\
\text { serrulatus (Fischer, } \\
\text { 1851) }\end{array}$ & $\begin{array}{l}\text { Cosm outside } \\
\text { S-Amer COL }\end{array}$ & $\begin{array}{l}\text { ant boy cun } \\
\text { mag }\end{array}$ & $0-4000$ & $\begin{array}{l}\text { ben co- } \\
\text { as-lag est } \\
\text { lit lak pl res } \\
\text { tan veg }\end{array}$ & $\begin{array}{l}\text { UARC } \\
\text { USNM }\end{array}$ & $\begin{array}{l}\text { Thiébaud, 1912; Reid, 1987; } \\
\text { Gaviria, 1988; Fuentes-Reinés } \\
\text { et al., } 2013\end{array}$ \\
\hline $\begin{array}{l}\text { Eucyclops titicacae } \\
\text { Kiefer, } 1957\end{array}$ & $\begin{array}{l}\text { BOL COL PER } \\
\text { VEN }\end{array}$ & lag & 0 & $\begin{array}{l}\text { ben co- } \\
\text { as-lag veg }\end{array}$ & UARC & $\begin{array}{l}\text { Fuentes-Reinés \& Suárez-Mo- } \\
\text { rales, } 2013\end{array}$ \\
\hline $\begin{array}{l}\text { Macrocyclops albidus } \\
\text { albidus (Jurine, 1820) }\end{array}$ & Cosm & $\begin{array}{l}\text { ant atl boy } \\
\text { cun mag met }\end{array}$ & $0-4100$ & $\begin{array}{l}\text { ben co- } \\
\text { as-lag res } \\
\text { lak lit pn } \\
\text { sw veg }\end{array}$ & UARC & $\begin{array}{l}\text { Thiébaud, 1912; Gaviria 1988; } \\
\text { Fuentes-Reinés et al., } 2013\end{array}$ \\
\hline $\begin{array}{l}\text { Macrocyclops albidus } \\
\text { principalis Herbst, } 1962 \\
\end{array}$ & BRA COL VEN & san mag & $0-1500$ & $\begin{array}{l}\text { cav co- } \\
\text { as-lag veg }\end{array}$ & UARC & $\begin{array}{l}\text { Petkovski, 1988; Fuentes-Rei- } \\
\text { nés et al., } 2013\end{array}$ \\
\hline $\begin{array}{l}\text { Paracyclops chiltoni } \\
\text { (Thompson, 1882) }\end{array}$ & Cosm & cun mag & $0-2700$ & $\begin{array}{l}\text { ben co- } \\
\text { as-lag lak } \\
\text { res riv }\end{array}$ & UARC & $\begin{array}{l}\text { Gaviria, 1988; Fuentes-Reinés et } \\
\text { al., } 2013\end{array}$ \\
\hline $\begin{array}{l}\text { Paracyclos fimbriatus } \\
\text { (Fischer, 1853) }\end{array}$ & Palearc COL & mag & 0 & $\begin{array}{l}\text { ben res lak } \\
\text { coas-lag } \\
\text { riv }\end{array}$ & MBUCV & $\begin{array}{l}\text { Fuentes-Reinés et al., 2013; Fu- } \\
\text { entes-Reinés \& Suárez-Morales, } \\
2015\end{array}$ \\
\hline $\begin{array}{l}\text { Tropocyclops prasinus } \\
\text { altoandinus Gaviria, } \\
1994\end{array}$ & COL & $\begin{array}{l}\text { ant cun boy } \\
\text { met }\end{array}$ & $\begin{array}{l}2000- \\
3775\end{array}$ & $\begin{array}{l}\text { ben lak lit } \\
\text { res }\end{array}$ & $\begin{array}{l}\mathrm{ICN}- \\
\mathrm{MHN}\end{array}$ & $\begin{array}{l}\text { Gaviria, 1994; Aranguren } \mathcal{E} \\
\text { Andrade, } 2003 \text { (as T. prasinus } \\
\text { prasinus); Aranguren-Riaño } \\
\text { et al., 2011; Aranguren-Riaño, } \\
2014\end{array}$ \\
\hline $\begin{array}{l}\text { Tropocyclops prasinus } \\
\text { prasinus (Fischer, 1860) }\end{array}$ & BRACOL & $\begin{array}{l}\text { ant atl boy } \\
\text { cun nar }\end{array}$ & $0-3670$ & $\begin{array}{l}\text { ben est lag } \\
\text { lit }\end{array}$ & USNM & $\begin{array}{l}\text { Reid, 1988; Aranguren-Riaño } \\
\text { et al., 2011; Aranguren-Riaño, } \\
\text { 2014; JM \& NA pers. obs. } 2016\end{array}$ \\
\hline \multicolumn{7}{|l|}{$\begin{array}{l}\text { SUBFAMILY HALI- } \\
\text { CYCLOPINAE }\end{array}$} \\
\hline $\begin{array}{l}\text { Halicyclops exiguus } \\
\text { Kiefer, } 1934\end{array}$ & $\begin{array}{l}\text { BRA COL CRI HTI } \\
\text { GUF }\end{array}$ & lag mag & 0 & $\begin{array}{l}\text { euryhal } \\
\text { coas-lag lit } \\
\text { veg }\end{array}$ & UARC & $\begin{array}{l}\text { Fuentes-Reinés \& Suárez-Mo- } \\
\text { rales, 2015; Fuentes-Reinés \& } \\
\text { Suárez-Morales, } 2018\end{array}$ \\
\hline $\begin{array}{l}\text { Halicyclops gaviriai } \\
\text { Suárez-Morales \& } \\
\text { Fuentes-Reinés, } 2014 \\
\end{array}$ & COL & lag & 0 & $\begin{array}{l}\text { cost-lag pel } \\
\text { veg }\end{array}$ & $\begin{array}{l}\text { ECO-CHZ } \\
\text { UARC }\end{array}$ & $\begin{array}{l}\text { Suárez-Morales \& Fuentes-Rei- } \\
\text { nés, } 2014\end{array}$ \\
\hline $\begin{array}{l}\text { Halicyclops hurlberti } \\
\text { Rocha, } 1991\end{array}$ & $\begin{array}{l}\text { COL MEX-si USA- } \\
\text { ca }\end{array}$ & mag & 0 & coas-lag & UARC & $\begin{array}{l}\text { Fuentes-Reinés \& Suárez-Mo- } \\
\text { rales, } 2018\end{array}$ \\
\hline $\begin{array}{l}\text { Halicyclops venezuela- } \\
\text { ensis Lindberg, } 1954\end{array}$ & $\begin{array}{l}\text { BLZ COL MEX } \\
\text { VEN }\end{array}$ & lag mag & 0 & $\begin{array}{l}\text { euryhal } \\
\text { coas-lag lit } \\
\text { veg }\end{array}$ & UARC & $\begin{array}{l}\text { Fuentes-Reinés et al., 2013; Fu- } \\
\text { entes-Reinés \& Suárez-Morales, } \\
\text { 2015, } 2018\end{array}$ \\
\hline \multicolumn{7}{|l|}{$\begin{array}{l}\text { FAMILY ERGASILI- } \\
\text { DAE }\end{array}$} \\
\hline $\begin{array}{l}\text { Ergasilus argulus Cres- } \\
\text { sey, } 1970\end{array}$ & COL & val & 0 & $\begin{array}{l}\text { host: Stron- } \\
\text { gylura flu- } \\
\text { viatilis } \mathcal{E} \text { S. } \\
\text { scapularis }\end{array}$ & $\begin{array}{l}\text { FMNH } \\
\text { USNM }\end{array}$ & Cressey \& Colette, 1970 \\
\hline
\end{tabular}




\begin{tabular}{|c|c|c|c|c|c|c|}
\hline $\begin{array}{l}\text { Ergasilus curticrus } \\
\text { Muriel-Hoyos, } \\
\text { Santana-Piñe- } \\
\text { ros,Cruz-Quintana \& } \\
\text { Suárez-Morales, } 2015\end{array}$ & COL & met & 300 & $\begin{array}{l}\text { host: } \mathrm{Bryco-} \\
\text { nops giaco- } \\
\text { pinii }\end{array}$ & $\begin{array}{l}\text { ECO-CHZ } \\
\text { IMCN }\end{array}$ & Muriel-Hoyos et al., 2015 \\
\hline $\begin{array}{l}\text { Ergasilus pitalicus } \\
\text { Thatcher, } 1984\end{array}$ & COL & val & $<900$ & $\begin{array}{l}\text { host: Cich- } \\
\text { lasoma sp. }\end{array}$ & USNM & Thatcher, 1984 \\
\hline $\begin{array}{l}\text { Paraergasilus longidigi- } \\
\text { tus Yin, } 1954\end{array}$ & $\begin{array}{l}\text { CHN COL MEX } \\
\text { USA }\end{array}$ & mag & 0 & lit veg & $\begin{array}{l}\text { MBUCV } \\
\text { UARC }\end{array}$ & Fuentes-Reinés et al., 2012 \\
\hline \multicolumn{7}{|l|}{$\begin{array}{l}\text { FAMILY KELLER- } \\
\text { IDAE }\end{array}$} \\
\hline $\begin{array}{l}\text { Kelleria reducta Suá- } \\
\text { rez-Morales \& Fuen- } \\
\text { tes-Reinés, } 2015 \text { (c) }\end{array}$ & COL MEX & lag & 0 & $\begin{array}{l}\text { coas-lag } \\
\text { pel }\end{array}$ & UARC & $\begin{array}{l}\text { Suárez.Morales \& Fuentes-Rei- } \\
\text { nés, 2015c }\end{array}$ \\
\hline \multicolumn{7}{|l|}{$\begin{array}{l}\text { FAMILY LERNAEI- } \\
\text { DAE }\end{array}$} \\
\hline $\begin{array}{l}\text { Lernaea cyprinacea } \\
\text { Linnaeus, } 1758\end{array}$ & Cosm & mag & 30 & $\begin{array}{l}\text { host: } \\
\text { Prochilodus } \\
\text { magdalenae }\end{array}$ & & Sarmiento \& Rodriguez, 2013 \\
\hline $\begin{array}{l}\text { Lernaea pirapitingae } \\
\text { (Thatcher \& Paredes, } \\
\text { 1985) }\end{array}$ & COL & met & 300 & $\begin{array}{l}\text { host: } \\
\text { Piaractus } \\
\text { brachypo- } \\
\text { mus }\end{array}$ & & Thatcher, 2000 \\
\hline \multicolumn{7}{|l|}{$\begin{array}{l}\text { FAMILY OITHONI- } \\
\text { DAE }\end{array}$} \\
\hline $\begin{array}{l}\text { Oithona amazonica } \\
\text { Burckhardt, } 1912\end{array}$ & $\begin{array}{l}\text { BRA COL GUF } \\
\text { VEN }\end{array}$ & vich & 300 & lak & & Rivera-Rondón et al., 2010 \\
\hline $\begin{array}{l}\text { Oithona oswaldocruzi } \\
\text { Oliveira, } 1945\end{array}$ & $\begin{array}{l}\text { COL BRA ECU } \\
\text { SLV HND PRI TTO } \\
\text { VEN }\end{array}$ & lag mag & 0 & $\begin{array}{l}\text { coas-lag } \\
\text { mg pel }\end{array}$ & UARC & $\begin{array}{l}\text { Fuentes-Reinés et al., 2013; Fu- } \\
\text { entes-Reinés-Suárez-Morales, } \\
2015\end{array}$ \\
\hline \multicolumn{7}{|l|}{$\begin{array}{l}\text { ORDER HARPACTI- } \\
\text { COIDA }\end{array}$} \\
\hline \multicolumn{7}{|l|}{ FAMILY AMEIRIDAE } \\
\hline $\begin{array}{l}\text { Nitokra affinis colom- } \\
\text { biensis Fuentes-Reinés } \\
\text { \& Suárez-Morales, } \\
2014 \text { (a) }\end{array}$ & $\mathrm{COL}$ & lag & 0 & $\begin{array}{l}\text { coas-lag } \\
\text { euryhal }\end{array}$ & $\begin{array}{l}\text { ECO-CHZ } \\
\text { UARC }\end{array}$ & $\begin{array}{l}\text { Fuentes-Reinés \& Suárez-Mora- } \\
\text { les, 2014a }\end{array}$ \\
\hline $\begin{array}{l}\text { Nitokra lacustris sinoi } \\
\text { Marcus \& Por, } 1961\end{array}$ & $\begin{array}{l}\text { CUB MEX USA-fl } \\
\text { COL ROU }\end{array}$ & mag lag & 0 & $\begin{array}{l}\text { euryhal } \\
\text { ben co- } \\
\text { as-lag mg } \\
\text { pel veg }\end{array}$ & $\begin{array}{l}\text { MBUCV, } \\
\text { UARC }\end{array}$ & $\begin{array}{l}\text { Fuentes-Reinés \& Zoppi de } \\
\text { Roa, 2013a; Fuentes-Reinés \& } \\
\text { Suárez-Morales, 2014b }\end{array}$ \\
\hline $\begin{array}{l}\text { Nitokra taylori Gómez, } \\
\text { Carrasco \& Mora- } \\
\text { les-Serna, } 2012\end{array}$ & SAF COL & lag & 0 & $\begin{array}{l}\text { coas-lag } \\
\text { pel }\end{array}$ & UARC & $\begin{array}{l}\text { Fuentes-Reinés \& Suárez-Mora- } \\
\text { les, 2014b }\end{array}$ \\
\hline \multicolumn{7}{|l|}{$\begin{array}{l}\text { FAMILY CANTHO- } \\
\text { CAMPTIDAE }\end{array}$} \\
\hline $\begin{array}{l}\text { Attheyella (Canthosella) } \\
\text { chocoensis Gaviria \& } \\
\text { Defaye, } 2012\end{array}$ & COL & cho & 50 & phytot & $\begin{array}{l}\text { ICN-MHN } \\
\text { MNHN } \\
\text { MNRJ }\end{array}$ & Gaviria \& Defaye, 2012 \\
\hline
\end{tabular}




\begin{tabular}{|c|c|c|c|c|c|c|}
\hline $\begin{array}{l}\text { Attheyella (Chappui- } \\
\text { siella) fuhrmanni (Thié- } \\
\text { baud, 1912) }\end{array}$ & $\begin{array}{l}\text { ARG BRA GTM } \\
\text { CRI COL URY } \\
\text { VEN }\end{array}$ & $\begin{array}{l}\text { ant atl cun } \\
\text { mag }\end{array}$ & $0-2600$ & $\begin{array}{l}\text { ben res lak } \\
\text { mm-pd } \\
\text { coas-lag sw }\end{array}$ & $\begin{array}{l}\text { MBUCV, } \\
\text { UARC }\end{array}$ & $\begin{array}{l}\text { Thiébaud, 1912; Chappuis, } \\
\text { 1956; Fuentes-Reinés \& Zoppi } \\
\text { de Roa, 2013a }\end{array}$ \\
\hline $\begin{array}{l}\text { Attheyella (Delachau- } \\
\text { xiella) freyi Löffler, } \\
1963\end{array}$ & COL ECU & cau & 3500 & ben pd & $\begin{array}{l}\text { MNHN } \\
\text { NHMW }\end{array}$ & $\begin{array}{l}\text { SG pers. obs. 2007; Gaviria \& } \\
\text { Defaye, } 2012\end{array}$ \\
\hline $\begin{array}{l}\text { Cletocamptus dominica- } \\
\text { nus Kiefer, } 1934\end{array}$ & Ant COL & mag & 20 & $\begin{array}{l}\text { coas lag pd } \\
\text { sa-wa veg }\end{array}$ & UARC & Gómez et al., 2017 \\
\hline $\begin{array}{l}\text { Cletocamptus helobius } \\
\text { Fleeger, } 1980\end{array}$ & MEX COL & lag & 0 & $\begin{array}{l}\text { coas-lag } \\
\text { mg euryhal }\end{array}$ & UARC & $\begin{array}{l}\text { Fuentes-Reinés \& Suárez-Mora- } \\
\text { les, 2014b }\end{array}$ \\
\hline $\begin{array}{l}\text { Cletocamptus nudus } \\
\text { Gómez, } 2005\end{array}$ & Neotr & lag mag & 0 & $\begin{array}{l}\text { coas-lag co- } \\
\text { ast-pd mg } \\
\text { euryhal }\end{array}$ & INV & $\begin{array}{l}\text { Fuentes-Reinés \& Suárez-Mo- } \\
\text { rales, 2014b; Fuentes-Reinés et } \\
\text { al., } 2015\end{array}$ \\
\hline $\begin{array}{l}\text { Cletocamptus samari- } \\
\text { ensis Fuentes-Reinés, } \\
\text { Zoppi de Roa \& Tor- } \\
\text { res, } 2015\end{array}$ & COL & mag & 0 & coas-pd & INV & Fuentes-Reinés et al., 2015 \\
\hline $\begin{array}{l}\text { Cletocamptus sinoalen- } \\
\text { sis Gómez, Fleeger, } \\
\text { Rocha-Olivares \& } \\
\text { Foltz, } 2004\end{array}$ & BRA COL MEX & mag & 0 & $\begin{array}{l}\text { coas-lag } \\
\text { ben }\end{array}$ & UARC & Fuentes-Reinés et al., 2018 \\
\hline $\begin{array}{l}\text { Elaphoidella grandidieri } \\
\text { (Guerne \& Richard, } \\
\text { 1893) }\end{array}$ & Pantr & ant lag & $0-1600$ & $\begin{array}{l}\text { ben co- } \\
\text { as-lag pn } \\
\text { res veg }\end{array}$ & UARC & $\begin{array}{l}\text { SG pers. obs. 2007; Fuentes-Rei- } \\
\text { nés \& Zoppi de Roa, 2013b; } \\
\text { Fuentes-Reinés \& Suárez-Mora- } \\
\text { les, 2014b }\end{array}$ \\
\hline $\begin{array}{l}\text { Elaphoidella bidens } \\
\text { bidens (Schmeil, 1894) }\end{array}$ & Cosm & ant mag & $0-1000$ & $\begin{array}{l}\text { ben lak } \\
\text { coas-lag } \\
\text { veg }\end{array}$ & UARC & $\begin{array}{l}\text { SG pers. obs. 2007; Fuentes-Rei- } \\
\text { nés \& Zoppi de Roa, 2013a }\end{array}$ \\
\hline $\begin{array}{l}\text { Elaphoidella paramuna } \\
\text { Gaviria \& Defaye, } \\
2015\end{array}$ & COL & cun & 3500 & ben lak & $\begin{array}{l}\text { ICN-MHN } \\
\text { MNHN } \\
\text { NHMW }\end{array}$ & Gaviria \& Defaye, 2015 \\
\hline $\begin{array}{l}\text { Elaphoidella sewelli mi- } \\
\text { nuta (Chappuis, 1932) }\end{array}$ & $\begin{array}{l}\text { COL CAF REU } \\
\text { MDG }\end{array}$ & mag & 0 & $\begin{array}{l}\text { ben co- } \\
\text { as-lag veg }\end{array}$ & UARC & $\begin{array}{l}\text { Fuentes-Reinés \& Zoppi de } \\
\text { Roa, 2013a }\end{array}$ \\
\hline $\begin{array}{l}\text { Elaphoidella schubarti } \\
\text { Chappuis, } 1936\end{array}$ & $\begin{array}{l}\text { BRA COL ECU } \\
\text { VEN }\end{array}$ & mag & 4000 & $\begin{array}{l}\text { ben lak mo } \\
\text { pd }\end{array}$ & & Löffler, 1972 \\
\hline $\begin{array}{l}\text { Mesochra huysi Suá- } \\
\text { rez-Morales \& Fuen- } \\
\text { tes-Reinés, } 2015 \text { (a) } \\
\end{array}$ & COL & lag & 0 & $\begin{array}{l}\text { coas-lag lit } \\
\text { pel }\end{array}$ & $\begin{array}{l}\text { ECO-CHZ } \\
\text { UARC }\end{array}$ & $\begin{array}{l}\text { Suárez-Morales \& Fuentes-Rei- } \\
\text { nés, 2015a }\end{array}$ \\
\hline \multicolumn{7}{|l|}{$\begin{array}{l}\text { FAMILY CLETOD- } \\
\text { IDAE }\end{array}$} \\
\hline $\begin{array}{l}\text { Enhydrosoma lacunae } \\
\text { Jakuvisiak, } 1933\end{array}$ & Amer & lag & 0 & $\begin{array}{l}\text { coas-lag } \\
\text { mar mg } \\
\text { pel }\end{array}$ & UARC & $\begin{array}{l}\text { Fuentes-Reinés \& Suárez-Mora- } \\
\text { les, 2014b }\end{array}$ \\
\hline \multicolumn{7}{|l|}{$\begin{array}{l}\text { FAMILY ECTINOSO- } \\
\text { MATIDAE }\end{array}$} \\
\hline $\begin{array}{l}\text { Halectinosoma arangu- } \\
\text { reni Suárez-Morales } \\
\text { \& Fuentes-Reinés, } \\
2015 \text { (b) }\end{array}$ & $\mathrm{COL}$ & lag & 0 & $\begin{array}{l}\text { coas-lag lit } \\
\text { pel mg veg }\end{array}$ & $\begin{array}{l}\text { UARC } \\
\text { ECO-CHZ }\end{array}$ & $\begin{array}{l}\text { Suárez-Morales \& Fuentes-Rei- } \\
\text { nés, 2015b }\end{array}$ \\
\hline
\end{tabular}




\begin{tabular}{|c|c|c|c|c|c|c|}
\hline $\begin{array}{l}\text { Pseudobradya gascae } \\
\text { Suárez-Morales \& } \\
\text { Fuentes-Reinés, 2015b }\end{array}$ & COL & lag & 0 & $\begin{array}{l}\text { coas-lag } \\
\text { mg pel veg }\end{array}$ & $\begin{array}{l}\text { UARC } \\
\text { ECO-CHZ }\end{array}$ & $\begin{array}{l}\text { Suárez-Morales \& Fuentes-Rei- } \\
\text { nés, } 2015 b\end{array}$ \\
\hline \multicolumn{7}{|l|}{$\begin{array}{l}\text { FAMILY LAOPHON- } \\
\text { TIDAE }\end{array}$} \\
\hline $\begin{array}{l}\text { Quinquelaophonte } \\
\text { quinquespinosa (Sewell, } \\
\text { 1924) }\end{array}$ & Cosm & lag & 0 & $\begin{array}{l}\text { coas-lag } \\
\text { pel }\end{array}$ & UARC & $\begin{array}{l}\text { Fuentes-Reinés \& Suárez-Mora- } \\
\text { les, } 2014 b\end{array}$ \\
\hline \multicolumn{7}{|l|}{ FAMILY METIDAE } \\
\hline $\begin{array}{l}\text { Metis holothuriae (Ed- } \\
\text { wards, 1891) }\end{array}$ & Cosm & lag & 0 & $\begin{array}{l}\text { coas-lag } \\
\text { mg pel veg }\end{array}$ & UARC & $\begin{array}{l}\text { Fuentes-Reinés \& Suárez-Mora- } \\
\text { les, } 2014 b\end{array}$ \\
\hline \multicolumn{7}{|l|}{$\begin{array}{l}\text { FAMILY MIRACII- } \\
\text { DAE }\end{array}$} \\
\hline $\begin{array}{l}\text { Sarsamphiascus hirtus } \\
\text { (Gurney, 1927) }\end{array}$ & Eur COL BRA NZL & lag & 0 & $\begin{array}{l}\text { coas-lag } \\
\text { mar pel } \\
\text { veg }\end{array}$ & UARC & $\begin{array}{l}\text { Fuentes-Reinés \& Suárez-Mora- } \\
\text { les, } 2014 b\end{array}$ \\
\hline $\begin{array}{l}\text { Schizopera evelynae } \\
\text { Fuentes-Reinés \& } \\
\text { Gómez, } 2014\end{array}$ & COL & lag & 0 & $\begin{array}{l}\text { coas-lag } \\
\text { mg pel }\end{array}$ & $\begin{array}{l}\text { UARC, } \\
\text { EMUCOP }\end{array}$ & Fuentes-Reinés \& Gómez, 2014 \\
\hline $\begin{array}{l}\text { Schizopera knabeni } \\
\text { Lang, } 1965\end{array}$ & $\begin{array}{l}\text { COL Gulf-Mex Pac } \\
\text { USA }\end{array}$ & lag & 0 & $\begin{array}{l}\text { coas-lag } \\
\text { sa-wa pel } \\
\text { veg }\end{array}$ & UARC & $\begin{array}{l}\text { Fuentes-Reinés \& Suárez-Mora- } \\
\text { les, } 2014 \text { b }\end{array}$ \\
\hline $\begin{array}{l}\text { Robertsonia propinqua } \\
\text { (Scott T., 1894) }\end{array}$ & Cosm & lag & 0 & $\begin{array}{l}\text { coas-lag } \\
\text { pel }\end{array}$ & UARC & $\begin{array}{l}\text { Fuentes-Reinés \& Suárez-Mora- } \\
\text { les, } 2014 b\end{array}$ \\
\hline \multicolumn{7}{|l|}{$\begin{array}{l}\text { FAMILY PARAST- } \\
\text { ENOCARIDIDAE }\end{array}$} \\
\hline $\begin{array}{l}\text { Colombocaris isabellae } \\
\text { Gaviria, Defaye \& } \\
\text { Corgosinho, } 2017\end{array}$ & COL & boj cun & 3000 & $\begin{array}{l}\text { ben lak mo } \\
\text { semiter }\end{array}$ & ICN-MHN & $\begin{array}{l}\text { Gaviria et al., 2017; SG \& DD } \\
\text { pers. obs. } 2016\end{array}$ \\
\hline $\begin{array}{l}\text { Noodtcaris columbiensis } \\
\text { (Noodt, 1972) }\end{array}$ & COL & met & 300 & int-riv & DZMB & $\begin{array}{l}\text { Noodt, } 1972 \text { (as Parastenocaris } \\
\text { columbiensis); Gaviria et al., } 2017\end{array}$ \\
\hline $\begin{array}{l}\text { Noodtcaris kubitzkii } \\
\text { (Noodt, 1972) }\end{array}$ & COL & met & 300 & int-riv & DZMB & $\begin{array}{l}\text { Noodt, } 1972 \text { (as Parastenocaris } \\
\text { kubitzkii); Gaviria et al., } 2017\end{array}$ \\
\hline $\begin{array}{l}\text { Noodtcaris roettgeri } \\
\text { (Noodt, 1972) }\end{array}$ & COL & met & 300 & int-riv & DZMB & $\begin{array}{l}\text { Noodt, } 1972 \text { (as Parastenocaris } \\
\text { roettgeri); Gaviria et al., } 2017\end{array}$ \\
\hline \multicolumn{7}{|l|}{$\begin{array}{l}\text { FAMILY TACHIDI- } \\
\text { DAE }\end{array}$} \\
\hline $\begin{array}{l}\text { Euterpina acutifrons } \\
\text { (Dana, 1847) }\end{array}$ & Cosm & lag & 0 & $\begin{array}{l}\text { coas-lag } \\
\text { veg pel }\end{array}$ & UARC & $\begin{array}{l}\text { Fuentes-Reinés \& Suárez-Mora- } \\
\text { les, 2014b }\end{array}$ \\
\hline \multicolumn{7}{|l|}{$\begin{array}{l}\text { FAMILY TEGAST- } \\
\text { IDAE }\end{array}$} \\
\hline $\begin{array}{l}\text { Parategastes herteli } \\
\text { Jakobi, } 1953\end{array}$ & BRA COL & lag & 0 & $\begin{array}{l}\text { coas-lag } \\
\text { pel veg }\end{array}$ & UARC & $\begin{array}{l}\text { Fuentes-Reinés \& Suárez-Mora- } \\
\text { les, } 2014 \text { b }\end{array}$ \\
\hline
\end{tabular}


Abbreviations: World distribution: Amer - America, Ant - Antilles, ARG - Argentina, Atl - Atlantic Ocean, BLZ - Belize, BOL - Bolivia, BRA - Brazil, CAN - Canada, CAR - Caribbean Sea, CAF - Central African Republic, CHL - Chile, CHN - China, COL - Colombia, Cosm - cosmopolitan, CRI - Costa Rica, CUB Cuba, ECU Ecuador, SLV - El Salvador, Eur - Europe, GUF - French Guyana, GTM - Guatemala, Gulf-Mex - Gulf of Mexico, HTI - Haiti, Hisp - Hispaniola, HND - Honduras, Ind - Indian Ocean, Indo-Pac - IndoPacific Ocean, REU - La Réunion, Les-Ant - Lesser Antilles, MDG - Madagascar, MEX - Mexico, MEX-si - Mexico (Sinaloa), Med - Mediterranean Sea, Neotr - Neotropical region, NIC - Nicaragua, NZL - New Zealand, PRY - Paraguay, Pac - Pacific Ocean, Palearc - Palearctic region, Pantr - pantropical, PER Peru, PAN - Panama, PRI - Puerto Rico, ROU - Romania, S-Amer - South America, SAF - South Africa, Subtrop - subtropical, TTO - Trinidad and Tobago, Trop - tropical, URY - Uruguay, USA - United States of America, USA-ca - United States of America (California), USA-fl - United States of America (Florida), VEN Venezuela. Distribution in Colombia (Departments): ama (Amazonas), ant (Antioquia), atl (Atlántico), boy (Boyacá), cal (Caldas), cau (Cauca), cho (Chocó), cor (Córdoba), cund (Cundinamarca), guai (Guainía), hui (Huila), lag (La Guajira), ces (Cesar), mag (Magdalena), met (Meta), nar (Nariño), nsan (Norte de Santander), san (Santander), tol (Tolima), val (Valle del Cauca), vich (Vichada). Habitat in Colombia: coast-lag - coastal lagoon, backw - backwater of a river, ben - benthos, cav - cave, cie - "ciénaga" (=freshwater lagoon), coaslag - coastal lagoon, coas-lag-fw - coastal lagoon/freshwater zone, coas-pd - coastal pond, de - demersal, epib - epibenthic, est - "estero" (typical meadow in the east plains "Llanos"), estua - estuary, euryhal euryhaline, ig - "igapó" lake, int-riv - interstitial of a river, lak - lake, lit - littoral, mg - mangrove, mar - marine, mm-pd - man-made pond, mo - moss, pel - pelagic, phytot - phytotelmata, pl - plankton, pn - pond, res - reservoir, sa-wa - saltwater, semiter - semiterrestrial, sw - swamp, tan - water tank, tpl - treatment plant, var -“ varzea” lake, veg - macrophytes. Acronyms: DZMB - Deutsches Zentrum für Marine Biodiversitätsforschung, Senckenberg am Meer, Wilhemshaven, Germany; ECO-CHZ - Collection of Zooplankton at El Colegio de la Frontera Sur, Chetumal, Mexico; EMUCOP - Copepoda collection of the Instituto de Ciencias del Mar y Limnología, Mazatlán Marine Station, Sinaloa, Mexico; FMNH - Field Museum of Natural History, Chicago, Ill., USA; ICN-MHN - CR - Museo de Historia Natural - Crustacean Colección - Instituto de Ciencias Naturales at Universidad Nacional de Colombia, Bogotá, Colombia; IMCN - Zoological Collection of Scientific References, Departmental Museum of Natural Sciences Federico Carlos Lehmann Valencia, Cali, Colombia; INV Museum Instituto de Investigaciones Marinas INVEMAR, Santa Marta, Colombia; MBUCV - Museo de Biología de la Universidad Central de Venezuela, Crustacean Section, Caracas, Venezuela; MNHN-Muséum Nationale d'Histoire Naturelle, Paris, France; MNRJ - Museo Nacional, Universidade Federal do Rio de Janeiro, Brazil; NHMW - Naturhistorisches Museum Wien, Austria; UARC - Museo de Colecciones Biológicas at the Universidad del Atlántico, Barranquilla, Colombia; UIS Universidad Industrial de Santander, Colección Limnológica; USNM - U.S. National Museum, Washington, USA. Bibliographic reference: pers. obs. - personal observation, DB - D. Baribwegure, DD - D. Defaye, JM - J. Molano, NA - N. Aranguren, SG - S. Gaviria. 
In the Neotropical region as a whole, the number of copepods living in inland waters comprises 458 species and subspecies. That value is nearly four times as high as that in Colombia (Table 2).

Table 2. Comparative taxonomic synopsis of the families, genera and subgenera of the copepods reported in continental water bodies of Colombia until 2007 (Gaviria \& Aranguren, 2007) and until 2018 (present inventory), and their representation in the Neotropical region. Numbers indicates species for each genus (numbers of subspecies are indicated in brackets). Numbers in bold indicate total number of species and subspecies for each order. * Parasitic genera, **Ergasilus comprises 20 species in South America and Mexico, and an unknown (?) number of species in Central America and the Antillean Islands. ${ }^{* *}$ Species of Parastenocaris (2007) were allocated to the new genus Noodtcaris.

\begin{tabular}{|c|c|c|c|}
\hline Taxa & Neotropics & $\begin{array}{l}\text { Colombia reports } \\
\text { until } 2007\end{array}$ & $\begin{array}{l}\text { Colombia reports } \\
\text { until } 2018\end{array}$ \\
\hline Order Calanoida & 110 & 14 & 21 \\
\hline \multicolumn{4}{|l|}{ Family Acartiidae } \\
\hline \multicolumn{4}{|l|}{ Acartia } \\
\hline Acanthacartia & 3 & 1 & 1 \\
\hline Odontacartia & 2 & & 1 \\
\hline \multicolumn{4}{|c|}{ Family Centropagidae } \\
\hline Boeckella & 15 & 2 & 2 \\
\hline \multicolumn{4}{|c|}{ Family Diaptomidae } \\
\hline \multicolumn{4}{|l|}{ Arctodiaptomus } \\
\hline Arctodiaptomus & 1 & 1 & 1 \\
\hline Colombodiaptomus & $1(2)$ & $1(2)$ & $1(2)$ \\
\hline Dactylodiaptomus & 1 & & 1 \\
\hline Dasydiaptomus & 1 & & 1 \\
\hline Notodiaptomus & 36 & 6 & 7 \\
\hline Prionodiaptomus & 2 & 1 & 1 \\
\hline Rhacodiaptomus & $8(2)$ & 1 & 1 \\
\hline \multicolumn{4}{|l|}{ Family Lucicutidae } \\
\hline Lucicutia & 23 & & 1 \\
\hline \multicolumn{4}{|c|}{ Family Pseudodiaptomidae } \\
\hline Pseudodiaptomus & 12 & & 1 \\
\hline \multicolumn{4}{|l|}{ Family Temoridae } \\
\hline Temora & 4 & & 1 \\
\hline Order Cyclopoida & 208 & 41 & 61 \\
\hline
\end{tabular}




\section{Family Corycaeidae}

Corycaeus

Corycaeus

\section{Family Cyclopidae}

\section{Subfamily Cyclopinae}

\begin{tabular}{|c|c|c|c|}
\hline Acanthocyclops & 4 & 1 & 1 \\
\hline Apocyclops & 5 & 2 & 2 \\
\hline Diacyclops & 10 & 2 & 2 \\
\hline Hesperocyclops & 2 & 1 & 1 \\
\hline Mesocyclops & $21(6)$ & 5 & 7 \\
\hline Metacyclops & $25(4)$ & $4(5)$ & $4(5)$ \\
\hline Microcyclops & $13(4)$ & 5 & $5(2)$ \\
\hline Neutrocyclops & 1 & 1 & 1 \\
\hline Thermocyclops & 8 & 2 & 4 \\
\hline \multicolumn{4}{|c|}{ Subfamily Eucyclopinae } \\
\hline Ectocyclops & 6 & 2 & 2 \\
\hline Eucyclops & $24(5)$ & 7 & 8 \\
\hline Macrocyclops & $3(5)$ & $1(2)$ & $1(2)$ \\
\hline Paracyclops & 8 & 3 & 4 \\
\hline Tropocyclops & $19(13)$ & $1(2)$ & $1(2)$ \\
\hline \multicolumn{4}{|c|}{ Subfamily Halicyclopinae } \\
\hline Halicyclops & 19 & & 4 \\
\hline \multicolumn{4}{|l|}{ Neocyclops } \\
\hline Protoneocyclops & 4 & 1 & 1 \\
\hline \multicolumn{4}{|l|}{ Family Ergasilidae } \\
\hline Ergasilus & $20+? * *$ & & 3 \\
\hline Paraergasilus & 1 & & 1 \\
\hline \multicolumn{4}{|l|}{ Family Kelleriidae } \\
\hline Kelleria & 1 & & 1 \\
\hline
\end{tabular}




\begin{tabular}{|c|c|c|c|}
\hline \multicolumn{4}{|l|}{ Family Lernaeidae } \\
\hline Lernaea & 3 & & 2 \\
\hline \multicolumn{4}{|l|}{ Family Oithonidae } \\
\hline Oithona & 5 & & 2 \\
\hline Order Harpacticoida & 140 & 14 & 39 \\
\hline \multicolumn{4}{|l|}{ Family Ameiridae } \\
\hline Nitokra & $9(6)$ & $1(1)$ & $4(3)$ \\
\hline \multicolumn{4}{|c|}{ Family Canthocamptidae } \\
\hline \multicolumn{4}{|l|}{ Attheyella } \\
\hline Canthosella & 8 & & 1 \\
\hline Chappuisiella & $17(2)$ & 2 & 2 \\
\hline Delachauxiella & $23(2)$ & 1 & 1 \\
\hline Cletocamptus & $15(2)$ & & 5 \\
\hline Elaphoidella & $35(3)$ & 5 & $8(1)$ \\
\hline Epactophanes & 1 & 1 & 1 \\
\hline Mesochra & 4 & & 1 \\
\hline \multicolumn{4}{|l|}{ Family Cletodidae } \\
\hline Enhydrosoma & 2 & & 1 \\
\hline \multicolumn{4}{|c|}{ Family Ectinosomatidae } \\
\hline Halectinosoma & 1 & & 1 \\
\hline Pseudobradya & 1 & & 1 \\
\hline \multicolumn{4}{|l|}{ Family Laophontidae } \\
\hline Quinquelaophonte & 1 & & 1 \\
\hline \multicolumn{4}{|l|}{ Family Metidae } \\
\hline Metis & 1 & & 1 \\
\hline
\end{tabular}




\begin{tabular}{|c|c|c|c|}
\hline \multicolumn{4}{|l|}{ Family Miraciidae } \\
\hline Sarsamphiascus & 2 & & 1 \\
\hline Schizopera & $8(2)$ & & 2 \\
\hline Robertsonia & 3 & & 1 \\
\hline \multicolumn{4}{|l|}{ Family Parastenocarididae } \\
\hline Colombocaris & 1 & & 1 \\
\hline Noodtcaris & 4 & $3^{* * *}$ & 3 \\
\hline \multicolumn{4}{|l|}{ Family Phyllognathopodidae } \\
\hline Phyllognathopus & $2(1)$ & 1 & 1 \\
\hline \multicolumn{4}{|l|}{ Family Tachididae } \\
\hline Euterpina & 1 & & 1 \\
\hline \multicolumn{4}{|l|}{ Family Tegastidae } \\
\hline Parategastes & 1 & & 1 \\
\hline Total species and subspecies & 458 & 69 & 121 \\
\hline Total freshwater copepods & 328 & 68 & 82 \\
\hline $\begin{array}{l}\text { Total brackish water copepods (incl. marine species and } \\
\text { brackish water parasites) }\end{array}$ & 90 & 5 & 39 \\
\hline Total without parasitic copepods & 434 & 69 & 115 \\
\hline Parasitic copepods & $24+? * *$ & 0 & 6 \\
\hline
\end{tabular}

\section{Discussion}

The increase in copepod diversity presented here for Colombia reflects the exploration of new territories and new biotopes as well as taxonomic changes in the subclass Copepoda during the past 11 years. Thirteen species new to science have been described from Colombia since 2007.

The number of brackish taxa (including marine species collected in brackish environments) found in coastal lagoons and temporal ponds near the coast, reached 39 species and subspecies. The number of copepods parasitic on fish has risen to six species. This number is expected to increase when the Amazonian region is surveyed; it has not yet been studied for ergasilids and lernaeids.

Calanoid copepod numbers have been enriched by the discovery of the family Pseudodiaptomidae (Pseudodiaptomus marshi) and one additional species of Acartiidae (Acartia (Odontacartia) lilljeborgi) in coastal lagoons. Two additional families, Lucicutidae (Lucicutia flavicornis) and Temoridae (Temora turbinata), typical of marine waters, were also found in these environments. 
L. flavicornis is an oceanic species that sometimes invades neritic water (Vives \& Shmeleva, 2006). A coastal lagoon is not a typical habitat for that species and the two specimens found in the Ciénaga Grande de Santa Marta (Fuentes-Reinés et al., 2013) possibly arrived there with tidal currents. The same holds true for the presence of T. turbinata in the coastal lagoon Navío Quebrado (Fuentes-Reinés \& Suárez-Morales, 2015) because the species is a typical neritic-oceanic species (Vives \& Shmeleva, 2006).

In freshwaters, three additional species of the family Diaptomidae were recorded, two in the Amazon (Dactylodiaptomus pearsei and Notodiaptomus linus) (Aranguren, 2014) and one in the Orinoco basin (Dasydiaptomus coronatus) (Rivera-Rondón et al., 2010). Within calanoids, Notodiaptomus is the genus with the highest species number in Colombia (7), and is also the genus with the highest species richness in South America (Perbiche-Neves et al., 2014). The distribution of two species of the genus, Notodiaptomus dilatatus and N. echinatus, expanded to the Vichada department in the Orinoco basin (Rivera-Rondón et al., 2010). Additionally, Notodiaptomus simillimus and $N$. coniferoides increased their distribution to Córdoba in the Caribbean region (Villabona-González et al., 2011. Jaramillo-Londoño \& Aguirre-Ramírez, 2012; Aranguren, 2014).

Notodiaptomus henseni was newly registered in Córdoba and Boyacá. In Boyacá, the species was found in a high mountain lake (Laguna Verde, Páramo de Pisba, $2740 \mathrm{~m}$ a. s. 1.) (Aranguren \& Molano, pers. obs. 2014). This represents the second record of a species of Notodiaptomus in mountain waterbodies of the Andes. Recently, Alonso et al. (2017) described Notodiaptomus cannarensis from a water reservoir in southern Ecuador, located at $2127 \mathrm{~m}$ a. s. 1 .

Notodiaptomus coniferoides has a wide distribution in South America, ranging from the Amazon River to the mouth of the Paraná River (Perbiche-Neves et al., 2013; Previatelli et al., 2013). In Colombia it has been recorded at the interandean Magdalena valley and in the Caribbean region (Gaviria \& Aranguren, 2007). Specimens from Venezuela identified by Dussart (1984) as $N$. coniferoides should be referred to $N$. simillimus, a species very similar to the former (Cicchino et al., 2001). It is possible that some Colombian records of $N$. coniferoides correspond to $N$. simillimus, as is apparently also the case in northern Brazil (Previatelli et al., 2013).

Notodiaptomus maracaibensis is the species of the genus with the widest distribution in the Colombian Caribbean region. It was found together with three other species of the genus (Notodiaptomus henseni, N. coniferoides, N. simillimus) in the Ciénaga de Ayapel, Córdoba, where it reached the highest abundances among the planktonic copepods (Villabona-González et al., 2011). High densities of N. maracaibensis were also observed in the ciénaga-complex of Malambo, near the Magdalena River (Atencio et al., 2005). In the Lago de Maracaibo, Venezuela (locus typicus of the species), its populations are thought to be threatened. Due to its distribution and populations size in Colombia, its vulnerable status at the IUCN Red List (Baillie \& Groombridge, 1996) should be re-evaluated (Reid, pers. com. to SG).

Four species of Diaptomidae, Rhacodiaptomus ringueleti, Notodiaptomus dilatatus, Notodiaptomus linus and Notodiaptomus echinatus, seem to be restricted to lakes and rivers east of the Cordillera (Dussart, 1984; Cicchino \& Dussart, 1991; Rivera-Rondón, 2010; Aranguren, 2014), whereas records of $N$. simillimus stem from the same region and also from the Caribbean plains (Cicchino et al, 2001; SG, pers. obs. 2007; VillabonaGonzález et al., 2011).

Arctodiaptomus dorsalis is also widely distributed in Colombia, newly registered in Córdoba (Aranguren, 2014) as well as in Santander (Reid, 2007) and Norte de Santander in the Andean Cordillera (VillabonaGonzález et al., 2007). This species has an apparent center of origin in the lowlands around the Gulf of Mexico, Central America, the Greater Antilles and northern South America (Reid, 2007). The latter author discussed the increase of the species' distribution further north in the United States and further south in Colombia, influenced by human activities such as aquaculture and by colonisation of suitable eutrophic waterbodies. New records in the Caribbean region 
(Ciénaga de Ayapel) (Aranguren, 2014) and an Andean reservoir (Laguna Acuarela, Norte de Santander) (Villabona-González et al., 2007) in Colombia point to an expansion trend to the south.

Prionodiaptomus colombiensis is also widely distributed and was recorded for the first time in Cordoba (Álvarez, 2010) in the Caribbean region. Together with $N$. henseni, it is the only diaptomid copepod distributed from lowland waterbodies up to Andean lakes with an altitude of 2600-2800 $\mathrm{m}$ a. s. 1 .

Colombodiaptomus brandorffi, formely known from the paramo lakes of Cundinamarca, was also recorded from Laguna de Iguaque in Boyacá (SG pers. obs. 2010; Aranguren, 2014). No additional records of the cold stenothermic centropagids (Boeckella) were registered.

With 20 new taxa recorded in Colombia, the order Cyclopoida now reaches 61 species and subspecies. Two families, i.e. Kelleridae and Ergasilidae, formerly belonging to the order Poecilostomatoida, are now allocated in the order Cyclopoida. Khodami et al. (2017) recently demonstrated that the poecilostomatoid lineage lies within the latter order. Thus, Kelleria reducta and four ergasilid species were added to the list of cyclopoids.

Other nomenclatural changes have occurred in this order. Mesocyclops venezolanus Dussart, 1984 is no longer accepted and is now recognised as a junior synonym of Mesocyclops brasilianus Kiefer, 1933, according to Gutiérrez-Aguirre et al. (2006). The cyclopoid copepod Microcyclops alius (Kiefer, 1935) is a junior synonym of Microcyclops dubitabilis Kiefer, 1934 (Gutiérrez-Aguirre \& Cervantes-Martínez, 2016).

The exploration of coastal lagoons has also contributed to the increase in the species number of other cyclopoids for the country. Various brackish-water species now form part of the inventory. The genus Halicyclops, with four species (H. exiguus, $H$. gaviriai, $H$. hurlberti, $H$. venezuelaensis), was found in the plankton of brackish environments (Fuentes-Reinés et al., 2013; SuárezMorales \& Fuentes-Reinés, 2014; Fuentes-Reinés \&
Suárez-Morales, 2015, 2018). Another new family, Oithonidae, contributed with one new species (Oithona oswaldocruzi) collected in coastal lagoons (Fuentes-Reinés et al., 2013; Fuentes-Reinés \& Suárez-Morales, 2015). The species O. amazonica was found in freshwaters of the Orinoco basin (Rivera-Rondón, 2010). Corycaeus clausi (family Corycaeidae) was registered for the first time in a coastal lagoon in La Guajira department (FuentesReinés \& Suárez-Morales, 2015).

Some typical freshwater species were recorded in coastal lagoons. They were apparently collected in the freshwater areas of the lagoons and constitute new species for Colombia: Eucyclops titicacae and Paracylops fimbriatus (Eucyclopinae), as well as Mesocyclops ellipticus and Microcyclops anceps pauxensis (Cyclopinae) (FuentesReinés et al., 2013; Fuentes-Reinés \& Suárez-Morales, 2013, 2015). Other freshwater species, i.e. Ectocyclops rubescens, E. phaleratus, Macrocyclops albidus albidus, M. albidus principalis and Paracyclops chiltoni (found in the Ciénaga Grande de Santa Marta), are new for the department of Magdalena (Fuentes-Reinés et al., 2013).

The Cyclopinae Thermocyclops minutus, registered in Caribbean and Orinoco waterbodies (Alvarez, 2010; Rivera-Rondón et al., 2010), as well as Thermocyclops crassus from Caribbean and Amazonean lakes (Aranguren, 2014), are also new for the country. Collado et al. (1984) and Reid (1989) mentioned that most published records of $T$. crassus in South and Central America and the Caribbean region should be referred to $T$. decipiens, and that the only confirmed record of $T$. crassus is from Costa Rica. T. crassus has a cosmopolitan distribution outside the Neotropical region. Therefore, new records of T. crassus in Colombia should be accepted with caution. The species Thermocyclops tenuis extended its distribution to the departments Córdoba and Magdalena in the Caribbean region. Thermocyclops decipiens, with records in four new departments, is now present in 13 departments of Colombia. It is probably the most common Thermocyclops species in the country, as it was also mentioned for the neotropics (Reid, 1989). The subspecies Tropocyclops prasinus prasinus and T. prasinus altoandinus were found for the first time in the department of Boyacá (Aranguren, 2014; NA \& JM pers. obs. 2018). 
Eucyclops serrulatus has been recorded from several localities of Colombia. Nevertheless, and according with Alekseev et al. (2006), E. serrulatus is a Palearctic species. Records in America may be from introduced populations or even represent as yet undescribed species (Mercado-Salas et al., 2012). The redescription of E. serrulatus and other six species included characters not considered in the past that helped to discriminate the species: pore signature of the cuticula, ornamentation of the antennal basis and of the intercoxal sclerite of the fourth pair of legs (Alekseev et al., 2006). In a study of Eucyclops-species from Mexico, Mercado-Salas \& Suárez-Morales (2014) redescribed four of them and considered that most of the remaining species of the genus in the Neotropical region should be redescribed. Eight species of the genus are now known from Colombia, but that number is probably an understimation.

As parasitic copepods are now also considered in the inventory, we have listed six species belonging to the cyclopoid family Ergasilidae (4) and Lernaeidae (2). Two ergasilids have been found in fish inhabiting rivers in southwestern Colombia (Ergasilus argulus and E. pitalicus), one in the Eastern Plains in the Meta departement (E. curticrus) and one in the Ciénaga Grande de Santa Marta (Paraergasilus longidigitus). The family Lernaeidae is represented by Lernaea pirapitingae from fish of the Meta River (Thatcher, 2000) and by Lernaea cyprinacea. The latter species was introduced to Colombia with fish (Carassius auratus and Cyprinus carpio) used in aquaculture, and it has been also recorded in Trichogaster microlepis (Rodríguez Gómez, 1981). AlvaradoForero \& Gutiérrez-Bonilla (2002) argued that this parasitic copepod is widespread in the country. It was also found recently as parasite of Prochilodus magdalenae from a fish farm in Gaira, department of Magdalena (Sarmiento \& Rodríguez, 2013). In Mexico, three species of ergasilids and L. cyprinacea have been reported in freshwater fish (Morales-Serna et al., 2012). Most of the parasitic copepods in South America have been recorded from the Amazon and the northeastern region of Brazil. In the Brazilian Amazon, 14 species of Ergasilus and 2 members of
Lernaea have been recorded (Muriel-Hoyos et al., 2015; Luque \& Tavarés, 2007). This means that at least ergasilids can be expected also as fish ectoparasites in the Colombian Amazon.

The order Harpacticoida showed the highest increase in species richness (2007: 14, 2018: 39). Within the 25 new species recorded for the country, 10 are new to science. Newly described species with the locus typicus in Colombia were Nitokra affinis colombiensis (Ameiridae), Attheyella (Canthosella) chocoensis, Cletocamptus nudus, Cletocamptus samariensis, Elaphoidella paramuna, Mesochra huysi (Canthocamptidae), Halectinosoma arangureni, Pseudobradya gascae (Ectinosomatidae), Schizopera evelynae (Miraciidae), and Colombocaris isabellae (Parastenocarididae).

The intensive taxonomic research on harpacticoids from the coastal lagoons Ciénaga Grande de Santa Marta (Fuentes-Reinés et al., 2013; Fuentes-Reinés \& Zoppi de Roa, 2013a, 2013b; Suárez-Morales \& Fuentes-Reinés, 2015a; Fuentes-Reinés et al., 2018) and Laguna Navio Quebrado (Fuentes-Reinés \& Gómez, 2014; Fuentes-Reinés \& Suárez-Morales, 2014a, 2014b; Suárez.Morales \& Fuentes-Reinés, 2015b), from two temporal ponds in the Caribbean region (FuentesReinés et al., 2015; Gómez \& Fuentes-Reinés, 2017), from water bodies in the páramo (Gaviria \& Defaye, 2012, Gaviria et al., 2017a, 2017b), and phytotelmata from the rain forest (Gaviria \& Defaye, 2012) explains this increase in species numbers.

Most harpacticoid copepods are typical inhabitants of benthic environments. The benthic families recorded in Colombia are Ameiridae, Canthocamptidae, Cletodidae, Ectinosomatidae Laophontidae, Metidae, Parastenocarididae, Tachididae and Tegastridae. Few families of the order have worldwide representatives in the plankton of coastal lagoons. In Colombia, only the family Miraciidae is known in this environment, represented by Schizopera (two species), Sarsamphiascus and Robertsonia (each with one species).

A revision of the columbiensis-group of Noodt (1972) from the family Parastenocarididae led Gaviria et al. 
(2017a, 2017b) to propose a new genus (Noodtcaris) for Parastenocaris columbiensis, Parastenocaris kubitzkii and Parastenocaris roettgeri together with a Brazilian species. As no other species of Parastenocaris has been recorded in Colombia, the genus is no longer part of the Colombian copepod fauna.

Three freshwater canthocamptid copepods (Elaphoidella bidens bidens, Elaphoidella grandidieri, Atheyella (Chappuisiella) fuhrmani) already known from Colombia were recorded from coastal lagoons (Fuentes-Reinés \& Zoppi de Roa, 2013a, 2013b; Fuentes-Reinés \& Suárez-Morales, 2014b), probably collected in their freshwater zone. The species Attheyella (Chappuisiella) pichilafquensis Löffler, 1962, also registered in Colombia, is considered by some authors (Löffler, 1962, 1963; Gaviria \& Aranguren, 2007) to be an independant species, and by others (Reid, pers. com. to SG) a synonym of A.(Ch.) fuhrmanni (Thiébaud, 1912). This calls for restudying the comparative morphology of both species. Elaphoidella schubarti was recorded by Löffler (1972) in Colombia, collected in high mountain waterbodies of the Sierra Nevada de Santa Marta.

The significant proportion of copepod species recorded in lowland waterbodies $(74 \%)$ compared within highland regions (17\%) indicates a high heterogeneity of ecological conditions in this area. Nine percent of the species are distributed in both regions.

Concerning the presence of copepods in the Colombian departments, 8 of the 32 departments (Arauca, Caldas, Caquetá, Casanare, Guaviare, Putumayo, Quindío and Vaupés), have no reports. Except Caldas and Quindío, the remaining departments are located in the Orinoco and Amazonas basins, where few surveys of aquatic invertebrates have been carried out. The departements with the highest number of records are Magdalena (42), La Guajira (34) and Cundinamarca (27), due to the expert taxonomists that have worked in these regions.

The diversity of copepods in Colombian continental waters, including brackish species of coastal lagoons and ponds is with 119 species, lower than in Mexico (159 species) (Suárez-Morales et al., 1998). Considering only freshwater taxa (82 species and subspecies), the diversity of copepods in Colombia is lower than in Brazil (200) (Reid, 1998; Rocha \& Botelho, 1998; Santos Silva, 1998; Previatelli \& Santos-Silva, 2007; PerbicheNeves et al., 2013; Silva \& Perbiche-Neves, 2016; Corgosinho et al., 2017), similar to Mexico (78) (SuárezMorales et al., 2000) and higher than in Venezuela (66) (Dussart, 1984), Cuba (56) (Collado et al., 1984) and Costa Rica (25) (Morales-Ramírez et al., 2014).

\section{Conclusions}

The list presented here contributes to a better understanding of the biodiversity of Copepoda in Colombia. As only two coastal lagoons and two coastal ponds in the Caribbean region were investigated, surveys in other brackish environments are expected to increase our knowledge about copepod diversity. Ground water environments, including the interstitial of rivers and lakes, continue to be virtually unstudied habitats for copepods. Species of parastenocaridids, canthocamptids, certain ameirids and cyclopoids should occur there. The further study of unexplored territories and poorly studied habitats like benthos of water bodies, ground waters and semiterrestrial biotopes should increase the number of copepods of the continental waters of Colombia.

\section{Aknowledgements}

Thanks go to J.M. Fuentes-Reinés (Santa Marta), S. Gómez (Mazatlán) and N.F. Mercado-Salas (Wilhelmshaven) for sending requested articles, to Daniele Defaye (Paris), Deo Baribwegure (Gent) and Jeison Molano (Tunja) for providing records based on personal observations, and to L.M. Vásquez and J. Gallo (Medellín) for the collection of part of the samples in Antioquia and Chocó. We thank two anonymous reviewers for their throughfull suggestions. M. Stachowitsch (University of Vienna) revised the English text. 


\section{References}

Alekseev V., Dumont, H. J., Pensaert, J., Baribwegure, D. \& Vanfleten, J. R. (2006). A redescription of Eucyclops (Fisher, 1851) (Crustacea: Copepoda: Cyclopoida) and some related taxa, with a phylogeny of the E. serrulatus-group. Zoologica Scripta, 35, 123-147.

Alonso, M., Santos-Silva, E. N. \& Jaume, D. (2017). A new species of Notodiaptomus from the Ecuadorian Andes (Copepoda, Calanoida, Diaptomidae). ZooKeys, 697, 59-71.

Alvarado-Forero, H. \& Gutiérrez-Bonilla, F. (2002). Especies hidrobiológicas continentales introducidas y trasplantadas y su distribución en Colombia. Santafé de Bogotá, Colombia: MinAmbiente / RAMSAR / CVC.

Álvarez, J. P. (2010). Caracterización limnológica de las Ciénagas de Arcial, El Porro y Cintura (Río San Jorge) y Bañó, Charco Pescao y Pantano Bonito (Río Sinú), Departamento de Córdoba. In J.O. Rangel-Ch. (Ed.). Colombia Diversidad biótica IX: Ciénagas de Córdoba: Biodiversidad - Ecología y manejo ambiental. Pp: 509-556. Bogotá, Colombia: Universidad Nacional de Colombia, Instituto de Ciencias Naturales.

Aranguren, N. (1998). Estudio de los copépodos planctónicos (Crustacea) de la Ciénaga de Guarinocito, Magdalena Medio. (Tesis de maestría). Bogotá, D.C.: Universidad de Los Andes.

Aranguren-Riaño, N. (2014). Diversidad ecológica de crustáceos planctónicos en sistemas lénticos colombianos. (Tesis de Doctorado en Ciencias). Bogotá, D.C.: Universidad Nacional de Colombia, Facultad de Ciencias, Departamento de Biología.

Aranguren, N. \& Andrade, C. (2003). Estructura de la comunidad de zooplancton (Copépodos y Cladóceros) de tres lagunas Colombianas de alta montaña. Ciencia en Desarrollo (Revista de la Facultad de Ciencia, Universidad Pedagógica y Tecnológica de Colombia, Tunja), 1(1), 70-83.

Aranguren-Riaño, N., Guisante, C. \& Ospina, R. (2011). Factors controlling crustacean zooplankton species richness in Neotropical lakes. Journal of Plankton Research, 33(8), 1295-1303.

Aranguren-Riaño, N. J. \& Monroy-González, J. D. (2014). Respuesta del zooplancton en un sistema tropical
(Embalse La Chapa, Colombia) con alta tensión ambiental. Acta Biológica Colombiana, 19(2), 281-290.

Atencio F., L., Gutiérrez M., L. \& Gaviria M., S. (2005). Copépodos planctónicos del complejo cenagoso de Malambo (Atlántico, Colombia) y su relación con algunos factores físicos y químicos del agua. Revista Dugandia, Nueva Época, 1(2), 17-37.

Baillie, J. \& Groombridge, B. (Eds.) (1996). 1996 IUCN Red List of Threatened Animals. Gland, Switzerland \& Cambridge, UK: International Union for Conservation of Nature. 378 pp.

Buitrago, L. F. (1998). Dinámica poblacional de Arctodiaptomus dorsalis en un ecosistema tropical raso Laguna Parque Norte, Medellín, Colombia. (Tesis de maestría). Popayán: Universidad del Cauca.

Chappuis, P. A. (1956). Freilebende Ruderfusskrebse (Crustacea, Copepoda), II. Harpacticoida. In Gessner, F. \& Vareshi, V. (Eds.), Ergebnisse der Deutschen Limnologischen Venezuela-Expedition 1952, 1, 269-276.

Cicchino, G. \& Dussart, B. H. (1991). Una nueva especie del género Rhacodiaptomus (Crustacea Copepoda) de Venezuela. Acta Biológica Venezolana, 13(1-2), 105-108.

Cicchino, G., Santos Silva, E. N. \& Robertson, B. (2001). A new species of Notodiaptomus Kiefer, 1936 (Copepoda, Diaptomidae) from the Amazon and Orinoco River Basins. Hydrobiologia, 453/454, 539-548.

Cicchino, G., Zoppi de Roa, E. \& Montiel, E. (1989). Notodiaptomus henseni Dahl (Crustacea-Copepoda): un problema de sinonimia en el zooplancton de Venezuela. Acta Biológica Venezolana, 12(3-4), 98-105.

Collado, C., Fernando, C. H. \& Sephton, D. (1984). The freshwater zooplankton of Central America and the Caribbean. Hydrobiologia, 113, 25-67.

Corgosihno, P. H. C., Schizas, N. V., Previattelli, D., Rocha, C. E. F. \& Santos-Silva, E. N. (2017). A new genus of Parastenocarididae (Copepoda, Harpacticoida) from the Tocantins River basin (Goiás, Brazil), and a phylogenetic analysis of the Parastenocaridinae. Zoosystematics and Evolution, 93(1), 167-187.

Cressey, R. F. \& Colette, B. B. (1970). Copepods and needlefishes: A study in host-parasite relationships. Fishery Bulletin, 68(3), 347-432.

Dussart, B. H. (1984). Some Crustacean Copepoda from Venezuela. Hydrobiologia, 113, 105-119. 
Fuentes-Reinés, J. M., Gómez, S. \& Dorado-Roncancio, E. F. (2018). Record of Cletocamptus sinaloensis (Copepoda: Harpacticoida: Canthocamptidae) from the Caribbean coast of Colombia. Boletin Científico Museo de Historia Natural Universidad de Caldas, 22(2), 172-182.

Fuentes-Reinés J. M. \& Suárez-Morales, E. (2013). First record of the freshwater copepod Eucyclops titicacae Kiefer, 1957, new rank (Copepoda, Cyclopoida) in Colombia. Biota Neotropica, 13(4), 1-8.

Fuentes-Reinés J. M. \& Suárez-Morales, E. (2014a). A new subspecies of Nitokra affinis Gurney, 1927 (Copepoda, Harpacticoida) from the Caribbean coast of Colombia. Zookeys, 6(378), 1-15.

Fuentes-Reinés J. M., Suárez-Morales, E. (2014b). Annoted checklist and new records of Harpacticoida (Copepoda) from a coastal system of northern Colombia, South America. Crustaceana, 87(2), 212-255.

Fuentes-Reinés J.M. \& Suárez-Morales, E. (2015). Checklist of planktonic copepoda from a Colombian coastal lagoon with record of Halicyclops exiguus Kiefer. Boletín de Investigaciones Marinas y Costeras, 44(2), 369-389.

Fuentes-Reinés J. M. \& Suárez-Morales, E. (2018). Notes on Halicyclops (Copepoda, Cyclopoida, Cyclopidae) from Colombia and the western Caribbean: a new record with a key to species of Group "B" sensu Rocha (1991). Revista Peruana de Biología, 25(2), 141-146.

Fuentes-Reinés, J. M. \& Zoppi de Roa, E. (2013a). Harpacticoid copepods from Ciénaga Grande de Santa Marta, Colombia. Metodos en Ecología y Sistemática, 8(3), 5-28.

Fuentes-Reinés, J. M. \& Zoppi de Roa, E. (2013b). Occurence of Elaphoidella grandidieri (Guerne \& Richard, 1893) (Crustacea: Copepoda: Harpacticoida) in Ciénaga Grande de Santa Marta, Colombia. Check List, 9(6), 1580-1583.

Fuentes-Reinés, J. M., Zoppi de Roa, E. \& Piñango, H. (2012). Redescription of Paraergasilus longidigitus Yin, 1954 (Copepoda: Ergasilidae) and report of its presence in South America. Métodos en Ecología y Sistemática, 7(3), 1-10.

Fuentes-Reinés, J. M., Zoppi de Roa, E. \& Torres, R. (2013). Calanoida and Cyclopoida (Copepoda: Crustacea) from Ciénaga Grande de Santa Marta, Colombia. Métodos en Ecología y Sistemática, 8(2), 54-103.
Fuentes-Reinés, J.M., Zoppi de Roa, E. \& Torres, R. (2015). A new species of Cletocamptus Schmankewitsch, 1875 (Crustacea, Copepoda, Harpacticoida) and the description of the male of $C . n u-$ dus from Colombia. Pan-American Journal of Aquatic Sciencies, 10(1), 1-18.

Fuentes-Reinés, J.M. \& Gómez, S. (2014). A new species of Schizopera (Copepoda: Harpacticoida: Miraciidae) from Colombia. Revista Científica Facultad de Medicina y Zootecnia de la Universidad de Córdoba, 19(3), 4199-4213.

Gallo-Sánchez, L. J., Aguirre-Ramírez, N. J., Palacio-Baena, J. A. \& Ramírez-Restrepo, J. J. (2009). Zooplancton (Rotífera y Microcrustacea) y su relación con los cambios de nivel del agua en la Ciénaga de Ayapel, Cordoba, Colombia. Caldasia, 3(2), 339-353.

Gaviria, S. (1988). Calanoida und Cyclopoida (Crustacea, Copepoda) der Cordillera Oriental der kolumbianischen Anden, mit besonderer Berücksichtigung ihrer Taxonomie, Ökologie und geographischen Verbreitung. (Dissertation), Wien: Universität Wien, Formal- und Naturwissenschaftliche Fakultät. 221 pp.

Gaviria, S. (1989). The calanoid fauna (Crustacea, Copepoda) of the Cordillera Oriental of the Colombian Andes. Hydrobiologia, 178, 113-134.

Gaviria, S. (1994) Los copépodos (Arthropoda, Crustacea) de vida libre de las aguas continentales de Colombia. Revista de la Academia Colombiana de Ciencias Exactas, Físicas y Naturales, 19(73), 361-385.

Gaviria, S. \& Aranguren, N. (2007). Especies de vida libre de la subclase Copepoda (Arthropoda, Crustacea) en aguas continentales de Colombia. Biota $\mathrm{Co}^{-}$ lombiana, 8(1), 53-68.

Gaviria, S. \& Defaye, D. (2012). A new species of Attheyella (Canthosella) from Colombia and redescription of Attheyella (Delachauxiella) freyi (Copepoda: Harpacticoida: Canthocamptidae). Zootaxa, 3179, 1-38.

Gaviria, S. \& Defaye, D. (2015). Description of Elaphoidella paramuna n.sp. (Canthocamptidae), a new harpacticoid copepod from Colombia. Crustaceana, 88(9), 1003-1029.

Gaviria, S., Defaye, D. \& Corgosinho, P. H. C. (2017a). Two new parastenocaridid genera (Copepoda, Harpacticoida) from South America, including a revision of Noodt's co- 
lumbiensis-group and the redescription of Parastenocaris tapajoensis Noodt, 1963. Crustaceana, 90(5), 535-570.

Gaviria, S., Defaye, D. \& Corgosihno, P.H.C. (2017b). Errata for "Two parastenocaridid genera (Copepoda, Harpacticoida) from South America, including a revision of the Noodt's columbiensis-group and the redescription of Parastenocaris tapajoensis Noo$\mathrm{dt}, 1963$. Crustaceana, 90(6), 765-766.

Gómez, S., Gerber, E. \& Fuentes-Reinés, J.M. (2017). Redescription of Cletocamptus albuquerquensis \& C. dominicanus (Harpacticoida: Canthocamptidae incertae sedis), and description of two new species from US Virgin Islands and Bonaire. Zootaxa, 4272(3), 301-359.

Gutiérrez-Aguirre, M. A. \& Cervantes-Martínez, A. (2016). Centropagidae. Taxonomic evaluation of eleven species of Microcyclops Claus, 1893 (Copepoda, Cyclopoida) and description of Microcyclops inarmatus sp. n. from America. Zookeys, 603, 33-69.

Gutiérrez-Aguirre, M. A., Suárez-Morales, E., Cervantes-Martínez, A., Elías-Gutiérrez, M. \& Previatelli, D. (2006). The neotropical species of Mesocyclops (Copepoda, Cyclopoida): an upgraded identification key and comments on selected taxa. Journal of Natural History, 40(9-10), 549-570.

Huys, R. \& Boxshall, G. A. (1991). Copepod Evolution. London, UK: The Ray Society.

Jaramillo-Londoño, J. C. \& Aguirre-Ramírez, N. J. (2012). Fluctuación de los ensambles planctónicos en la Ciénaga de Ayapel (Córdoba-Colombia) durante un ciclo semanal. Revista Ingeniería Universidad de Medellín, 11(21), 63-75.

Kiefer, F. (1956). Freilebende Ruderfusskrebse (Crustacea, Copepoda). Calanoida und Cyclopoida. In Gessner, F. \& Vareshi, V. (Eds.) Ergebnisse der Deutschen Limnologischen Venezuela-Expedition 1952, 1, 233-268.

Khodami, S., McArthur, J. V., Blanco-Bercial, L. \& Martínez Arbizu, P. (2017). Molecular phylogeny and revision of copepod Orders (Crustacea: Copepoda). Scientific Reports, 7(1), 1-11.

Löffler, H. (1972). Contribution to the limnology of high mountain lakes in Central America. Internationale Revue der gesamten Hydrobiologie und Hydrographie, 57(3), 397-408.
Luque J. L. \& Tavarés, L. E. R. (2007). Checklist of Copepoda associated with fishes from Brazil. Zootaxa, 1579, 1-39.

Mercado-Salas, N. F. \& Suárez-Morales, E. (2014). On Kiefer's American Eucyclops (Copepoda, Eucyclopinae): redescription and comments on the historical records of E. delachauxi, E. prionophorus, E. bondi and E. leptacanthus. ZooKeys, 402, 1-41.

Mercado-Salas, N. F., .Pozo, C., Morrone, J. J. \& Suárez-Morales, E. (2012). Distribution patterns of the American species of the freshwater genus Eucyclops (Copepoda: Cyclopoida). Journal of Crustacean Bio$\log y, 32(3), 457-464$.

Morales-Ramírez, A., Suárez-Morales, E., Corrales-Ugarte \& M. Esquivel-Garrote, O. (2014). Diversity of the free-living marine and freshwater Copepoda (Crustacea) in Costa Rica: a review. ZooKeys, 457, 15-33.

Morales-Serna, F.N., Gómez, S. \& Pérez-Ponce de León, G. (2012). Parasitic copepods reported from Mexico. Zootaxa, 3234, 43-68.

Muriel-Hoyos, F., Santana-Piñeros, A. M., Cruz-Quintana, Y. \& Suárez-Morales, E. (2015). A new species of Ergasilus Nordmann, 1832 (Copepoda: Cyclopoida: Ergasilidae) from Bryconops giacopinii Fernández-Yépez (Characidae) in the Vichada River Basin, Colombia. Sytematic Parasitology, 92(3), 241-249.

Noodt, W. (1972). Drei neue Parastenocaris aus Kolumbien (Crustacea, Copepoda). 1. Mitteilung über kolumbianische Grundwasser-Crustaceen. Studies on Neotropical Fauna \& Environment, 7, 101-112.

Pearse, A. S. (1915). An account of the Crustacea collected by the Walker Expedition to Santa Marta, Colombia. Proceedings of U.S. National Museum, 49, 531-556.

Perbiche-Neves, G., Boxshall, G. A., Paggi, J. C., Rocha, C. E. F., Previattelli, D. \& Nogueira, M. G. (2013). Two new species of Diaptomidae (Crustacea: Copepoda: Calanoida) from the Neotropical Region (Paraná River). Journal of Natural History, 39 pp.

Perbiche-Neves, G., Previattelli, D., Pie, M. R., Duran, A., Suárez-Morales, E., Boxshall G. A., Nogueira, M. G. \& Rocha, C. E. F (2014). Historical biogeography of the neotropical Diaptomidae (Crustacea: Copepoda). Frontiers in Zoology, 11(36), 1-8. 
Petkovski, T. K. (1988). Zur Cyclopidenfauna Kolumbiens (Crustacea, Copepoda). Acta Musei Macedonici Scientiarum Naturalium, 19(2/155), 39-64.

Previattelli, D., Perbiche-Neves, G. \& Santos-Silva, E. N. (2013). New Diaptomidae records (Crustacea: Copepoda: Calanoida: Diaptomidae) in the Neotropical Region. Check List, 9(4), 700-713.

Previattelli, D. \& Santos-Silva, E. N. (2007). A new Argyrodiaptomus (Copepoda: Calanoida: Diaptomidae) from the southwestern Brazilian Amazon. Zootaxa, 1518, 1-29.

Razouls C., Bovée, F. de, Kouwenberg, J. \& Desreumaux, N. (2005-2018). Diversity and Geographic Distribution of Marine Planktonic Copepods. Sorbonne Université, CNRS. Retrieved from https://copepodes.obs-banyuls.fr/en

Reid, J. W. (1988). Cyclopoid and harpacticoid copepods (Crustacea) from Mexico, Guatemala and Colombia. Transactions of the American Microscopical Society, 107, 190-202.

Reid, J. W. (1989). The distribution of species of the genus Thermocyclops (Copepoda, Cyclopoida) in the western hemisphere, with description of T. parvus, new species. Hydrobiologia, 175, 149-174.

Reid, J. W. (2007). Arctodiaptomus dorsalis: A case history of copepod dispersal. Banisteria, (Virginia $\mathrm{Na-}$ tural History Society), 30, 3-18.

Reid, J. W. (1998). Maxillopoda-Copepoda, Harpacticoida. In P.S. Young (Ed.), Catalogue of Crustacea of Brazil. Pp. 74-127. Rio de Janeiro, Brazil: Museo Nacional, Universidade Federal do Rio de Janeiro, III Serie, Serie Livros 6.

Reid, J. W., Molina, J. A. \& Fukushima, M. M. (1990). Metacyclops leptopus totaensis, new species from Lago de Tota, Colombia. Proceedings of the Biological Society of Washington, 103, 674-680.

Rivera-Rondón, C. A., Zapata, A. M., Pérez, D., Morales, Y., Ovalle, H. \& Álvarez, J. P. (2010). Caracterización limnológica de humedales de la planicie de inundación del río Orinoco (Orinoquia, Colombia). Acta Biológica Colombiana, 15(1), 145-166.

Rocha, C. E. F. \& Botelho, M. J. C. (1998). Maxillopoda-Copepoda, Cyclopoida. In Young, P. S. (Ed.). Catalogue of Crustacea of Brazil. Pp. 129-166. Rio de Ja- neiro, Brazil: Museo Nacional, Universidade Federal do Rio de Janeiro, III Serie, Serie Livros 6.

Rodríguez-Gómez, H. (1981). Parásitos piscícolas en aguas continentales de Colombia. Bogotá, Colombia: Ministerio de Agricultura. Inderena, Subgerencia de Pesca y Fauna Terrestre, Oficina de Comunicaciones. 35 pp.

Santos-Silva, E. N. (1998). Maxillopoda-Copepoda, Freshwater Calanoida. In Young, P. S. (Ed.) Catalogue of Crustacea of Brazil. Pp. 201-220. Rio de Janeiro, Brazil: Museo Nacional, Universidade Federal do Rio de Janeiro, III Serie, Serie Livros 6.

Sarmiento J. \& Rodríguez, A. (2013). Lerneosis en alevinos de Prochilodus magdalenae, Prochilodidae, cultivados en laboratorio. Revista Intrópica (Santa Marta), 8, 99-103.

Silva, W. M. \& Perbiche-Neves, G. (2016). Trends in freshwater microcrustaceans studies in Brazil between 1990 and 2014. Brazilian Journal of Biology, 77(3), 7.

Suárez-Morales, E., Reid, J. W. \& Gasca, R. (2000): Copepoda. In Llorente Bousquets, J. E., González Soriano, E. \& Papavero, N. (Eds.). Biodiversidad, Taxonomía y Biogeografía de Artrópodos de México: Hacia una Sintesis de su Conocimiento 2, D.R. Pp. 171-190. México D.F., México: Universidad Nacional Autónoma de México.

Suárez-Morales, E. \& Fuentes-Reinés, J. M. (2014). A new species of Halicyclops (Copepoda, Cyclopoida, Cyclopidae) from a lagoon system of the Caribbean coast of Colombia. Zookeys, 459, 35-47.

Suárez-Morales, E. \& Fuentes-Reinés, J. M. (2015a). A new species of Mesochra (Copepoda: Harpacticoida: Canthocamptidae) from a coastal system of northern Colombia with a key to the American species. Journal of Natural History, 49(45-48), 1-14.

Suárez-Morales, E. \& Fuentes-Reinés, J. M. (2015b). Two new species of ectinosomatid copepods (Harpacticoida: Ectinosomatidae) from the Caribbean coast of Colombia. Revista Mexicana de Biodiversidad, 86, 14-27.

Suárez-Morales, E. \& Fuentes-Reinés, J. M. (2015c). Record of Kelleria reducta (Copepoda: Poecilostomatoida: Kelleridae) from the Caribbean coast of Colombia. Revista Mexicana de Biodiversidad, 86, 28-33.

Thatcher, V. E. (1984). Ergasilus pitalicus, new species (Copepoda: Poecilostomatoida: Ergasilidae), a gill parasite of a cichlid fish from the Pacific coast of Colombia. Journal of Crustacean Biology, 4(3), 495-501. 
Thatcher, V. E. (2000). Perulernaea pirapitingae n.sp. (Copepoda: Lernaeidae) a parasite of the serrasalmid fish, Piaractus brachypomus from the Meta River, Colombia. Amazoniana, 16(1), 249-257.

Thiébaud, M. (1912). Copépodes de Colombie et des Cordillères de Mendoza. In O. Fuhrmann \& E. Mayor (Eds.), Voyage d'Exploration Scientifique en Colombie. Mémoires de la Societé Neuchâteloise de Sciencies Naturelles, 5, 160-175.

Torres, L. E. \& Rylander, K. (2006) Diversity and abundances of littoral cladocerans and copepods in nine Ecuadorian highland lakes. Revista de Biología Tropical, 54(1), 131-137.

Villabona-González, S. L., Aguirre R., N. J. \& Estrada P., A. L. (2011). Influencia de las macrófitas sobre la estructura poblacional de rotíferos y microscrustáceos en un plano de inundación tropical. Revista de Biología Tropical, 59(2), 853-870.
Villabona-González, S., Gavilán-Díaz, R. A. \& Estrada-Posada, A. L. (2007). Cambios nictemerales en la distribución vertical de algunos microcrustáceos en un lago artificial del neotrópico (Colombia). Limnética, 26(1), 75-88.

Villabona-González S. L., Ramírez-Restrepo, J. J., Palacio-Baena, J. A. \& Bonecker, C. C. (2015). Respuesta de la biomasa zooplanctónica a los gradientes de estado trófico y precipitación de un embalse tropical. Revista Academia Colombiana Ciencias Exactas, Fisicas y Naturales, 39(152), 374-388.

Vives, F. \& Shmeleva, A. A. (2006). Crustacea, Copépodos Marinos I. Calanoida. Fauna Ibérica vol. 29. Madrid, Spain: Museo Nacional de Ciencias Naturales, CSIC.

Walter, T. C. \& Boxshall, G. (2019). World of Copepods database. Retrieved from http:/ / www.marinespecies.org/copepoda on 2019-01-24

\section{Santiago Gaviria}

University of Vienna,

Department of Limnology and Bio-Oceanography.

Technisches Büro für Biologie Dr. Gaviria-Melo

Vienna, Austria

santiago.gaviria@gmx.at

https://orcid.org/0000-0002-5959-7919

\section{Nelson Aranguren-Riaño}

Universidad Pedagógica y Tecnológica de Colombia,

Unidad de Ecología en Sistemas Acuáticos UDESA

Tunja, Colombia

nelson.aranguren@uptc.edu.co

https://orcid.org/0000-0003-0117-1015

\section{Continental copepods (Crustacea: Hexanauplia) of} Colombia: revision and additions to the inventory

Citación del artículo: Gaviria, S. \& Aranguren-Riaño, N. (2019). Continental copepods (Crustacea: Hexanauplia) of Colombia: revision and additions to the inventory. Biota Colombiana, 20(1), 50-74. DOI: 10.21068/c2019. v20n01a04.

Recibido: 28 de septiembre de 2018

Aceptado: 30 de enero de 2019 